\title{
Stable Isotope Customer List and Summary of Shipments-FY 1977
}

W. C. Davis

\section{OAK RIDGE NATIONAL LABORATORY} OPERATED BY UNION CARBIDE CORPORATION - FOR THE DEPARTMENT OF ENERGY 


\section{DISCLAIMER}

This report was prepared as an account of work sponsored by an agency of the United States Government. Neither the United States Government nor any agency Thereof, nor any of their employees, makes any warranty, express or implied, or assumes any legal liability or responsibility for the accuracy, completeness, or usefulness of any information, apparatus, product, or process disclosed, or represents that its use would not infringe privately owned rights. Reference herein to any specific commercial product, process, or service by trade name, trademark, manufacturer, or otherwise does not necessarily constitute or imply its endorsement, recommendation, or favoring by the United States Government or any agency thereof. The views and opinions of authors expressed herein do not necessarily state or reflect those of the United States Government or any agency thereof. 


\section{DISCLAIMER}

Portions of this document may be illegible in electronic image products. Images are produced from the best available original document. 
Printed in the United States of America. Available from National Technical Information Service

U.S. Department of Commerce

5285 Port Royal Road, Springfield, Virginia 22161

Price: Printed Copy $\$ 4.50$; Microfiche $\$ 3.00$

This report was prepared as an account of work sponsored by an agency of the United States Government. Neither the United States Government nor any agency thereof, nor any of their employees, contractors, subcontractors, or their employees, makes any warranty, express or implied, nor assumes any legal liability or responsibility for any third party's use or the results of such use of any information, apparatus, product or process disclosed in llis report, nor represents that its use by such third party would not infringe privately owned rights. 
Contract No. W-7405-eng-26

CHEMICAL TECHNOLOGY DIVISION

STABLE ISOTOPE CUSTOMER LIST

AND SUMMARY OF SHIPMENTS - FY 1977

Compiled by

W. C. Davis

Date Published - April, 1978

\section{PITHEE MN ONLY'}

MATTOBS OF TMIS REPORT ARE ILLEGIBLE IE'

hes bests arodich from the best available congr io grermit the broadest possible avails abitity.

NOTICE This document contains information of a preliminary nature. It is subject to revision or correction and therefore does not represent a final report.

OAK RIDGE NATIONAL LABORATORY

Oak R1dge, Tennessee 37830 operated by UNION CARBIDE CORPORATION

for the

DEPARTMENT OF ENERGY 


\section{THIS PAGE}

\section{WAS INTENTIONALLY LEFT BLANK}


CONTENTS

$\underline{\text { Page }}$

INTRODUCTION

I. ALPHABETICAL LIST OF CUSTOMERS, WITH CONTACTS AND ISOTOPES PURCHASED $\ldots \ldots \ldots \ldots \ldots \ldots \ldots \ldots \ldots$

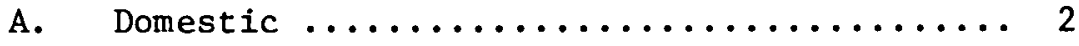

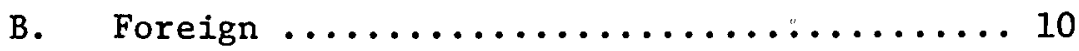

II. ALPHABETICAL LIST OF ISOTOPES, CROSS-REFERENCED TO CUSTOMER NUMBERS ........ 18

A. Domestic ...................... 18

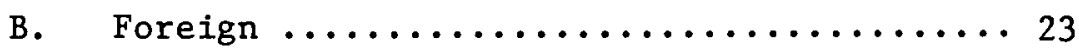

III. ALPHABETICAL LIST OF STATES AND COUNTRIES, CROSS-REFERENCED TO CUSTOMER NUMBERS ........ 29

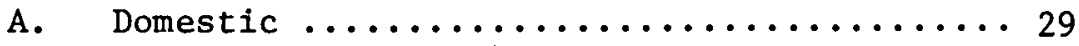

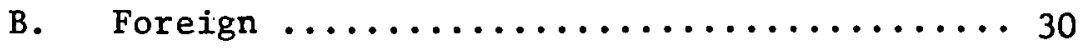

IV. STABLE ISOTOPE SALES - FY $1977 \ldots \ldots \ldots \ldots \ldots \ldots 31$ 
STABLE ISOTOPE CUSTOMER LIST

AND S SUMMARY OF SHIPMENTS - FY 1977

W. C. Davis

\section{INTRODUCTION}

This compilation is published as an aid to those concerned with the separation and sale of stable isotopes. The information is divided into four sections:

1. Alphabetical list of domestic and foreign customers, showing the stable isotopes purchased during the fiscal year;

2. Alphabetical list of isotopes, cross-referenced to customer numbers and divided into domestic and foreign categories;

3. Alphabetical list of states and countries, cross-referenced to customer numbers and indicating geographical concentrations of isotope users;

4. Tabulation of the shipments, quantities, and dollars for domestic, foreign, and project categories for each isotope, with the totals for loaned isotopes shown at the end of the table.

Other reports published in this series are:

$$
\begin{aligned}
& \text { ORNL/TM-1928 } \\
& \text { ORNL/TM-2038 } \\
& \text { ORNL/TM-2401 } \\
& \text { ORNI./TM-2747 } \\
& \text { ORNL/TM-3146 } \\
& \text { ORNL/TM-3543 } \\
& \text { ORNL/TM-3987 } \\
& \text { ORNL/TM-4693 } \\
& \text { ORNL/TM-5089 } \\
& \text { ORNL/TM-5853 }
\end{aligned}
$$

We wish to thank S. E. Curtis of the Business Systems Department of the ORGDP Computing Technology Center for his assistance in developing the tabulations. 
I. ALPHABETICAL LIST OF CUSTOMERS, HITH CONTACTS AND ISOTOPES PURCHASED

\section{A. DOMESTIC}

1 Abilene Christian College Box 8208

Abilene, Texas

$5 * f e$

2 Accu-iabs Research, Inc.

8170 Wegt 44 th Avenue

wheat RIdge, Colorado 80033

$183 \mathrm{~W}$

3 Aerojet Nuclear Company

550 second St reet

P. 0 . Box $1845^{\circ}$

Idaho Falls. Iaaho a.3anI

$10 \mathrm{~B}, 120 \mathrm{Ba}, 130 \mathrm{Ce}, 132 \mathrm{Gd}, 1384 \mathrm{a}, 6 \mathrm{LI}$,

is5 Nd, SS, iazw, 103U, 171Yb, 1/3yb

4 Al abama Power Company

P. U. Box 2641

Birmingham, Alabama 35291

rLi

5 Al abama, University of

Medical Center

1919 Seventh Avenue, South

Birminghat, Ala oama 35486

S7Fe, 6LI

6 Alaska, University of

Cullege, Alaska 99735

3051

7 Al drich Chomloal Cü̈pally. Ine.

940 West St. Paul Avenue

Mil wa ukee, Hisconsin 53233

ILi

8 Allied Chemlcal Corporation

Central Accounts Payable

P. Q. Rnx $2251 R$

Moritistown. N. J. 07960

$79 \mathrm{ar}, \mathrm{D}$

9 Alligegonoral Nuclear Bervices BNF P-O sborn Road ( P. O. Box 847 )

Barnwell. Bunth tarolina 29018

$155 \mathrm{Gd}, 106 \mathrm{Nd}, 150 \mathrm{Nd}$

10 Arjonne National Laboratory

9700 South Cass Avenue

Argonne, Illinols 604.39

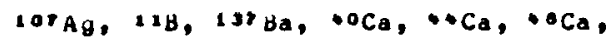

$63 \mathrm{Cu}, 65 \mathrm{Cu}, 160 \mathrm{Dy}, 5+\mathrm{Fe}, 100 \mathrm{Gd}$,

$30 \mathrm{~K}, 6 \mathrm{LI}, 7 \mathrm{LI}, 24 \mathrm{Mg}, 25 \mathrm{Mg}, 26 \mathrm{Mg}$,

$95 \mathrm{MO}, 97 \mathrm{MO}, 58 \mathrm{NL}, 60 \mathrm{NI}, 62 \mathrm{NI}$,

$1920 s, 200 \mathrm{~Pb}, 102 \mathrm{Pd}, 10 \circ \mathrm{Pd}, 100 \mathrm{Pd}$,

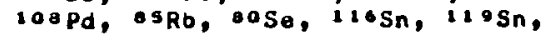

obsr, SS, Tgs, $205 T 1,172 y b, 902 \mathrm{r}$

$12 \mathbf{r}$
11 Arizona, University of

Post office Drawer 4100

Tucson, Arlzona 85721

$107 \mathrm{Ag}, 135 \mathrm{Ba}, 1+2 \mathrm{Ce}$, iscu, 156 Dy,

141DY, $167 \mathrm{Er}, 170 \mathrm{Er}, 153 \mathrm{Eu}, 155 \mathrm{Gd}$,

$170 \mathrm{HP}, 193 \mathrm{Ir}, 13 \% \mathrm{La}, 176 \mathrm{Lu}, 1+3 \mathrm{Nd}$,

$2000 \mathrm{~s}, 109 \mathrm{Sa}, 152 \mathrm{SA}$. $170 \mathrm{Yb}, 172 \mathrm{Yb}$, $672 n, 912 \mathrm{r}$

12 Arkansas Power and Light Company P. O. Box 551

Little Rock. Arkansas 72203

フレ

13 Atonerolic Chenetala Corporation 100 Falrchild Avenue Platinulou, N. Y. Lisos

ILI

14 Atomics International DIvision North Anerican Rockwell Corporation Post arflce Box 309 Canoga Park, Callfornia 91304

Tgs

15 Baltimore Gas and Electric Company Post orfice Box 1472

Baltimore, Haryland 21200

$7 \mathbf{L I}$

16 Dailul Regearch Corporation Swarthmore, Penngylvania 19081

$136 \mathrm{Ba}, 7 \mathrm{Ll}, 25 \mathrm{Mg}, 206 \mathrm{~Pb}, \mathrm{SS}$

17 Baylor Univergity

Box 6397

Waco, Taxas 76703

-LI, PLI

18 Ball Talephone Laboratortes Nurray Hill. New Jerooy 0797L

$10 \mathrm{~B}, 11 \mathrm{~B}, 19 \mathrm{Br}, 18 \mathrm{Br}, 57 \mathrm{Fe}, \operatorname{sefe}$

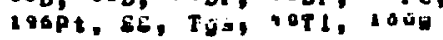

19 Brighaw Young Undroraily Provo, Utah 84601

$62 \mathrm{NI}$

20 British American Scientifle Instruments Corporatio 3937 Richaond Avenue Houston, Toxas 77027

$\because \mathbf{R} \mathbf{b}, \dot{S} \mathbf{r}$

21 Brookhaven National Laboratory Upton, Long Island, New York 11973

$10 \mathrm{~B}, 116 \mathrm{Cd}, 52 \mathrm{Cr}, 166 \mathrm{Er}, 120 \mathrm{Er}$, $6 \circ \mathrm{Ga}, 15 \circ \mathrm{Gd}, 1 \circ 0 \mathrm{Gd}, 12 \mathrm{Ge}, 7 \circ \mathrm{Ge}$,

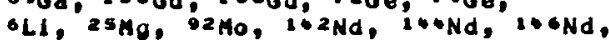
loNd, $130 \mathrm{Nd}, 4 N \mathrm{~N}, 1920 \mathrm{~s}, 20 \circ \mathrm{Pb}$, lospd, $10 \circ \mathrm{Pd}, 100 \mathrm{Pd}, 100 \mathrm{Pt}, 9 \circ \mathrm{Ru}$ rose, ise, zesl, i*sa, isosm, is2Si, $15 \circ S_{n}, 116 \mathrm{Sn}, 12 \circ \mathrm{Sn}, \mathrm{SS}, 120 \mathrm{Te}$, $130 \mathrm{Te}, \mathrm{Tgs,} \bullet 6 \mathrm{TI}, 6 \mathrm{Zn}, 00 \mathrm{Zr}, 02 \mathrm{Zr}$ 
22 Brookl yn College

Bedford Avenue and Avenue $H$

Brooklyn. New York 11210

-2Ca, 35Cl, 30K, 6Ll, 23Mg, 60NI, $2931, \operatorname{3os} 1$

23 Brown Univergity

Providence, Rhode Is I and 02912

$10 \mathrm{~B}$

24 California Institute of Technology $1201 \mathrm{E}$. Callfornia Boulevard

Pasadena, Californta 91109

${ }^{8} \mathrm{Ca}, 50 \mathrm{Cr}, 56 \mathrm{Fe}, 152 \mathrm{Gd}, 20 \mathrm{Mg}, 25 \mathrm{Mg}$, $26 \mathrm{Mg}, 62 \mathrm{NI}, 1000 \mathrm{~g}, 102 \mathrm{Pd}, 108 \mathrm{Pd}$, $120 \mathrm{Pd}, \mathrm{Tgs}, 0 \mathrm{Tl}, 160 \mathrm{w}, 160 \mathrm{Yb}$, $602 n$

25 Callifornla State College S1S1 State Colleye DrIve Los Angeles, California 90032

Q $\mathrm{RB}$

26 California, University of 400 Sproul Hall

Berkeley, Callfornia 94720

$113 \mathrm{Cd}, 20 \mathrm{~Pb}$

27 Cal ifornia, Universlty of Davis, California 95616

*Ca, *Ca, 7li, SS, 203TI, $205 \mathrm{TI}$

28 Callfornia, University of Post office Box 203

La Jolla, Calleornla 92037

$21 \mathrm{~d}, 135 \mathrm{Ba}, 162 \mathrm{Nd}, \mathrm{s} \mathrm{Sr}$

29 Callfornia, University of 405 Hilgard Avenue

Los Angeles, Callfornla 90024

$\bullet \mathrm{OCa}_{\text {, }} \mathrm{zCa}, \mathrm{SS}, \mathrm{Tg}$

30 Cal ifornia, Universlty of

900 Veteran Avenue

Los Angeles, Calleornia $\$ 0024$

$52 \mathrm{Cr}, 63 \mathrm{Cu}, 65 \mathrm{Cu}, 56 \mathrm{Fe}, 64 \mathrm{Nl}, 120 \mathrm{Te}$, - $0 \mathrm{Ti}$

31 Cal irornia, University of

Post ueflce Box 112

Adin Inigtretion huilding

Riverside, Californla 92502

$199 \mathrm{Hg}$

32 California, University of

San Francisco Medical Center

San Francisco, California 94122

-ocia, aca, 14JCd, erab

33 Californla, University of

Santa Barbara, Californla 93106

$3 \circ \mathrm{K}, 1 \mathrm{~K},>\mathrm{Li}, 20 \mathrm{~Pb}, 07 \mathrm{Rb}, 119 \mathrm{Sn}$
34 California, University of Lawrence Berkeley Laooratory Berkeley, Callfornla 94701

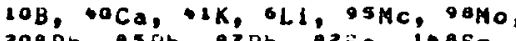

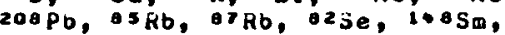
$130 \mathrm{Sm}, 152 \mathrm{Sm}, 12 \circ \mathrm{Sn}, \mathrm{SS}, \mathrm{Tgs}, 6 \mathrm{Ti}$, SOT I

35 Callfornia, Uni versity of Laurence Liveroore Laooratory Box 808

Livermore, California 94550

$14 \mathrm{~B}, 130 \mathrm{Ba}, 100 \mathrm{Ce}, 1+2 \mathrm{Ce}, 170 \mathrm{E} r, 50 \mathrm{fe}$, $56 \mathrm{Fe}, 24 \mathrm{Mg}, 25 \mathrm{Mg}, 26 \mathrm{Mg}, 2+8 \mathrm{Nd}, 50 \mathrm{Ni}$, $1060 \mathrm{~g}, 1870 \mathrm{~s}, 100 \mathrm{Os}, 100 \mathrm{Pd}, \mathrm{SR}$,

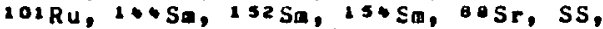
Tgs, IT०Yb

36 California, Uni versity of Los Alamos Scientifle Laboratory Post Urfice Box 1663 Los Alamos, New Mexico 87544

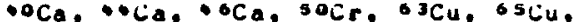
sofe, tsold, $191 \mathrm{I}, \mathrm{loj} \mathrm{Ir}, 39 \mathrm{~K}, 40 \mathrm{~K}$, 1K, $6 \mathrm{Li}, 7 \mathrm{LI}, 175 \mathrm{Lu}, 92 \mathrm{MO}, 166 \mathrm{Nd}$, $60 \mathrm{NI}, 61 \mathrm{NI}, 62 \mathrm{NI}, 64 \mathrm{NI}, 1860 \mathrm{~s}$, $1020 \mathrm{~s}, 200 \mathrm{~Pb}, 208 \mathrm{~Pb}, 192 \mathrm{Pt}, 100 \mathrm{Pt}$, $195 \mathrm{Pt}, 104 \mathrm{Pt}, 05 \mathrm{Rb}, 104 \mathrm{RU}, 20 \mathrm{SL}$, i2Sn, $116 \mathrm{Sn}, 120 \mathrm{Sn}, 0 \times \mathrm{Sr}, 86 \mathrm{Sr}$. $07 \mathrm{Sr}, \mathrm{SS}, 125 \mathrm{Te}, \mathrm{Tg}, * \mathrm{TI}, \bullet \mathrm{TI}$, SOT1, 203TL, 20sTl, $10 \cdot \mathrm{H}, 100 \mathrm{Yb}$, $\circ \mathrm{Zn}^{\circ}, 10 \mathrm{Zn}, 90 \mathrm{Zr}, 9 \mathrm{Zr}, 96 \mathrm{Zr}$

37 Carnegle Institution or Washington Washington, D. C. 20008

6LI, ILI

3 B Carnegle-Hellon University 5000 Forbes

Pittsburgh, Pennsylvania 25213

- $\mathrm{Ca}, 100 \mathrm{MO}, 1+6 \mathrm{Nd}, 10 \mathrm{BPd}, 1+45 \mathrm{~S}$, 15+S $116 \mathrm{Sn}, \mathrm{SS}, 6 \mathrm{Tl}, 17 \times \mathrm{Yb}$

39 Cathollc University of America 104 McCort-Ward Hall Washington, D. C. 20017

S6Fe, OLI

40 Chicago, University of 1160 East 55th Street Chicago, Lllinols 60615

$35 \mathrm{Cl}, 63 \mathrm{Cu}, 160 \mathrm{Er}, 1+4 \mathrm{Nd}, 204 \mathrm{~Pb}, \mathrm{SS}$

41 Cincinnati, Uni vergity of Cincinnati, Ohio 45221

${ }^{63} \mathrm{Cu}, 57 \mathrm{Fa}, 72 \mathrm{Ge}, 202 \mathrm{Hg}, 143 \mathrm{Nd}$, 10Nd, 110 Pd, $106 \mathrm{Pt}, 123 \mathrm{Te}, 128 \mathrm{Te}$ $130 \mathrm{Te}$

42 City or Hope Medical Center 1500 E. Duarte Road Duarte, Callfornia 91010

$42 \mathrm{Ca}$ 
43 Clark UnIversity

Worcester, Massachusetts 01610

$6 \mathrm{JCu}$

44 College of William and Mary

illiamsburg, Virginla 23185

$124 \mathrm{Te}, 125 \mathrm{Te}, 126 \mathrm{Te}, 4 \mathrm{TI}, \bullet 0 \mathrm{TI}, \bullet \mathrm{TI}$

45 Colorado School of Mines

Pust Ufflce Box 112

Golden, Colorado 80402

SLI, >LI

46 Colorado State University

Fort Conllina, Golorado a

7*ise

47 Colorado, Unluersity of

Boulder, Colorado 80302

* Ca, 1110d, 35Cl, s+fe, $190 \mathrm{Hg}$,

$200 \mathrm{Hy}, 202 \mathrm{Hg}, 20 * \mathrm{Hg}, 123 \mathrm{In}, 2 * \mathrm{Mg}$

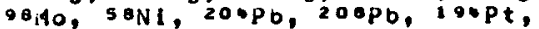

$100 \mathrm{pt}, \mathrm{BS} \mathrm{P}^{\circ}, \mathrm{Sr}, \mathrm{SS}, 122 \mathrm{Te}, 124 \mathrm{Te}$

$125 \mathrm{Te}, 130 \mathrm{Te}, \mathrm{THS}, 203 \mathrm{TI}, 205 \mathrm{TI}$,

962

48 Columbla University

Box 6, Low Librar

New York, New York 10027

$150 \mathrm{Nd}, \mathrm{TgS}, 182 \mathrm{U}$

49 Commercial Testiny and Engineer Ing Company Instrumental Analysis Division

14335 west $44 t h$ Avenue

Golden, Colorado 80401

us Ba, ${ }^{3} \mathrm{Ca}, \mathrm{s} * \mathrm{Fe}, 26 \mathrm{Mg}, 97 \mathrm{MO}, 117 \mathrm{Sn}$, $6 \mathrm{Ti}$

50 Connecticut, University of

Physical Sciences Bldg.

Storrs, Connect lcut 06268

$10 \mathrm{~g}, 56 \mathrm{Fe}, 61,25 \mathrm{Mg}, \mathrm{SS}$

51 Consolidated Edison

4 Irving Place

New Yurk, New York 10003

ILI

57. Fonnol l UnI versily

Ithaca, New York 14850

SBFe, $26 \mathrm{Mg}$

53 Corning Glass Work s

Houghton Park

Corning, New York 14830

$+2 \mathrm{Ca}, 50 \mathrm{Cr}, 53 \mathrm{Cr}, 5 * f e, 25 \mathrm{Mg}, 62 \mathrm{NI}$,

- TTI, SOTI

54 Cyclotron Corporation

950 Gi lan Street

Berkeley, Callfornia 94710

$\operatorname{Tg} \mathbf{s}$
55 DIsc Corporation

$7 \mathrm{Clarks} \mathrm{Hill}$ Avenue

Stanford, Connecticut 06902

$100 \mathrm{Cd}$

56 Dresser Atlas

Division of Drasser Industries, Inc.

P. O. Box 1407

Houston, Texas 77001

$10 \mathrm{~B}$

57 Du Pont de Nemours and Company, E. I. Savannah RIver Plant Alken, South Carolina 29801

-9K, IsaNd, 12,1

58 Duke Univoral ty

Durhaw. Narth Garolina 27Tud

$10 \mathrm{~B}, 12 \mathrm{~B}, \mathrm{CLI}^{\circ}, 95 \mathrm{HO}, 62 \mathrm{NL}, \mathrm{TgS}$,

$\bullet T i, 6+Z n, \bullet \bullet Z n$

59 Eayle-picher Industries, Inc.

Mlad, OkL ahoma 74354

'La

60 Eastman Kodak Company

Kodak Park Works

Rochester. Neu York 14650

$121 \mathrm{Cd}, 62 \mathrm{NI}, 207 \mathrm{~Pb}, 195 \mathrm{Pt}$

61 Efraton Callfornla, Inc.

3303 Harbor Bl ud.

Costa Mesa, California \$2626

QSRh, QTRb

62 Emory Uni versity

Atlanta, Georgla 30322

sife, SS, 6azn

63 Florida Power and Lignt Company

P. D. Bax 950

Miami. Florida 33101

TLI

64 Florida istate University

Tallahassee, Florida 32306

RIBr; $0 \mathrm{Ca},+\mathrm{Ca}, 1+2 \mathrm{CE}, 161 \mathrm{Dy}, 167 \mathrm{Er}$,

ISIEU, ISSGd, I76HR, $170 \mathrm{Lu}, 20 \mathrm{Hg}$,

$25 \mathrm{Hg}, 26 \mathrm{Mg}, 100 \mathrm{Mo}, 165 \mathrm{Nd}, 33 \mathrm{~S}, 28 \mathrm{Si}$.

$109 \mathrm{~S}, * \mathrm{TI}, \cdots \mathrm{Tl}, 17 \mathrm{Yb}, 6+2 \mathrm{Z}$.

$60 \mathrm{Zn}, 6 \cdot 2 \mathrm{Zn}$

65 Florida, Univorsity ur

Gainesuilie, Florida 32601

Tgs, $90 \mathrm{Zr}$

66 Ford Hotor Company

21500 Oakwood boulevard

Dearborn, Michlgan 48124

S7Fe 
67 General Electric Company

Post Ufrlce Box 254

San Jose, California 95103

$107 \mathrm{Ag}, 132 \mathrm{ba}$, socr, sofe, SS

68 General Electric Company

Development Center

P. 0. Box 8

Schenectady, New York 12301

$10 \mathrm{~B}$

69 Georye Mason University

10675 Lee Highway

Fal reax, Virginia 22030

BPK

70 Georgla Ingtltute of Technology

225 North Avenue, N..

Atlanta, Georgia 30332

$179 \mathrm{HE}, 150 \mathrm{Nd}, 100 \mathrm{Pd}, 110 \mathrm{Pd}, 173 \mathrm{Yb}$

71 Georgia State College

33 Gilmer Street, S. W.

Atlanta, Georgia. 30303

$120 \mathrm{Ca}, 180 \mathrm{He}$

72 Georgia, University of

Athens, Georgia 30601

Tgs

73 Gulf E'nergy and Environmental Systems

P. D. Box 608

San Diego, Californla 92112

$>$ L i

74 Har shaw Chealcal Company

Division of Kewanee 011 Company

6801 Cochran Road

Solion, Ohlo 44139

$6 \mathrm{Li}, \mathrm{TLI}$

75 Harvard Unluersity

Cambridge, Massachusetts 02138

$135 \mathrm{Ba}, \mathrm{SS}$

76 Herbert Lehoan College of the City University of New York

Hedford Park Boulevard West

Bronx, New York 10468

$128 \mathrm{Te}, 180 \mathrm{~W}, 66 \mathrm{Zn}$

77 Hewlett Packard Company

1501 Page MLll Road

Palo Alto, Calicornia 94304

$190 \mathrm{Hg}, 204 \mathrm{Hg}, 67 \mathrm{Rb}$, SS

7) Hope College

Holland, Michlgan 49423

$10 \mathrm{~B}$
79 Howard University

24016 th Street

Hashington, D. C. 20001

$11 \mathrm{Cd}$

80 Hughes Aircraft Company

Research Laboratorles

3011 Mal ibu Canyon Road

Mal ibu, Callfornila 90265

$90 R \mathbf{u}$

a1 ICN-NBCo

26201 Hiles Road

Claveland: Ohlo 44128

$130 \mathrm{Ba}, \mathrm{ISEU}$

82 Illinois, University of 203 Adminigtration Building $E$ Urbana, Illinols 61803

- ${ }^{\circ a, ~ 3 s c l, ~ S 0 f e, ~} 92 \mathrm{Mo}, 32 \mathrm{~S}, 33 \mathrm{~s}$, us, $>$, Se, SS, $\rightarrow \mathrm{Ln}$

83 Illinols, Uni versity of P. 0. Box 4348

Chlcago, Illinols 60080

$25 \mathrm{Mg}$

84 Ind lana Uni versity bloovington, Indlana 47401

$10 \mathrm{~B}, * 0 \mathrm{Ca}, 208 \mathrm{Cd}, 5 \times \mathrm{Fe}, 50 \mathrm{Fe}, 39 \mathrm{~K}$, +1K, $6 \mathrm{Li}, 26 \mathrm{Hg}, 30 \mathrm{Ni}, 60 \mathrm{Ni}, 62 \mathrm{Ni}$, 2OBPO, OSKb, BTRb, $20 \mathrm{Sl}, 30 \mathrm{SI}, \mathrm{SS}$, Tg9, $106 \mathrm{id}$

os Ind lava and Mi chigan Pouer Company Donald $c$. Cook Nuclear Plant P. O. Box 458

Bridguan, Michigan 49106

$T_{L L}$

46 Intel com Rad Tech

P. O. Box 80817

Jan Digje, Callifornta 92138

10B, OL

87 International Business Machlnes Corp. Post arflee Box 218

Yorktown Helghts, New York 10598

Sife

88 Iowa State Un Ivers!ty

Aces. Iow a 50010

$5 * F+20 * \mathrm{Hg}$

89 Ithaca College

Ithaca, Nat York 14850

STFe

9o Johns Hopking University Baltimore. Maryland 21218 
91 Kansas State University

Manhattan, Kansag 66502

$2 * M g, T g s$

92 Kentucky, University of Lexington, Kentucky 40506

$56 \mathrm{Fe}, 106 \mathrm{Nd}, 58 \mathrm{Ni}, 1860 \mathrm{~s}, 100 \mathrm{Sm}$, $1505 \mathrm{~m}, 152 \mathrm{~S}$, $126 \mathrm{~S}, \mathrm{SS}, \mathrm{Tg} g$

93 Loras College

Dubuque, Iowa 52001

${ }^{6}{ }^{\mathrm{Cu}},{ }^{\circ} \mathrm{C} \mathrm{Cu}, \mathrm{S} \bullet \mathrm{Fe}$

94 Louisiana State Unlversity Nuclear Srienco Conter

Baton Rouge, Loulsiana

70803

- ${ }^{\circ} \mathrm{ap}, \mathrm{H}, \mathrm{l}, \mathrm{B}+\mathrm{Sr}, \mathrm{Tg}$

$95 \mathrm{MDH}$ Indugtries, Inc. 426 . Duarte Road Monrovia, California 91016

$6 \mathrm{Li}$

96 Mallinckrodt Chemical Workg $3600 \mathrm{~N}$. Second Street St. Louls, Missouri 63160

$$
\text { 7* se }
$$

97 Maryland, University of College Park, Maryland 20742

$110 \mathrm{Cd}, 63 \mathrm{Cu}, 115 \mathrm{In}, 61.1,20 \mathrm{epb}$, $2851,240 \mathrm{Sm}, 150 \mathrm{Sm}, 132 \mathrm{Sm}, 134 \mathrm{Sm}, \mathrm{sS}$, $90 \mathrm{Zr}$

98 Magsachugetto General Hosplta! I ootupe Lauorazory

Boston, Massachusetts 02114

- oし́a, ss

99 Massacnusetts Inst itute of Techinology Box ôs

Cambr ldge. Massachusetts 02139

$135 \mathrm{Ba}, 237 \mathrm{Ba}, 13 \mathrm{ABa}, 167 \mathrm{Er}, 30 \mathrm{Fe}$, $125 \mathrm{In}, 39 \mathrm{~K}, 175 \mathrm{Lu}, 2 \times \mathrm{Mg}, 26 \mathrm{Mg}, 13 \mathrm{NA}$

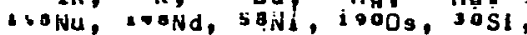
14०Sm, 140 Sm, Tgs, $182 \mathrm{~W}, 100 \mathrm{~W}, 170 \mathrm{Yb}$, $172 \mathrm{Yb}, 173 \mathrm{Yb}^{2}, 1+\mathrm{Y}_{0}$

100 Magdo husotts, Uill versity of Amherst. Massachusetts 01002

- $2 \mathrm{Ca}, \mathrm{SS}, \mathrm{Tg}$

101 Medical College or Wisconsin 8700 W. Hisconsin Avenuo Mll waukee, Wiscons in 53226

$113 \mathrm{Cd}, 12 \times \mathrm{Cd}, 63 \mathrm{Cu}, \operatorname{sis}, 6 r \mathrm{Zn}$

102 Medi-Physles, Inc.

5855 Christie Avenue

Emeryilie, California 94608

$112 \mathrm{Cd}, 122 \mathrm{Te}, 12+\mathrm{Te}, 68 \mathrm{Zn}$
103 Med I-Physics, Inc. 900 Durham Avenue South Plainfleld, New Jersey 07080

$122 \mathrm{Te}, 00 \mathrm{Zn}$

104 Miami University Oxford, Onlo 45056

ons r

105 Michigan State University

East Lansing, Michlyan 48823

$39 \mathrm{~K}, 142 \mathrm{Nd}, 14 \times \mathrm{Nd}, 33 \mathrm{~S}, 34 \mathrm{~S}, 40 \mathrm{Se}$, ozse, $2931, \operatorname{ses} 1,1183 \mathrm{n}, \mathrm{SS}$

106 Michlgan, University of Ann Arnor. Michigan 48104

35Cl, 37Cl, 656u, s7Fa, NoEn, $1225 n, 1205 n, 662 n, 672 n$

107 MicroMatter Company 197 - $34 \mathrm{th}$ Avenue East seattle, washington $\$ 3102$

$20 \mathrm{Mg}, 200 \mathrm{~Pb}, 150 \mathrm{Sa}$, is $2 \mathrm{Se}, 120 \mathrm{Sn}$, SS, Tgs, $92 \mathrm{Zr}$

108 Aicrotolis

F. 0. Box 283

Argonne, Illinois 60439

$200 \mathrm{~Pb}, 110 \mathrm{Sn}, 120 \mathrm{Sn}, \mathrm{SS}, \mathrm{T}_{\mathrm{g}}, 49 \mathrm{TI}$, $6,2 n, 672 n$

109 Ninnesota. University of 402 Morrill Hall Minneapolis, Minnesota 55455

23*Ba, $136 \mathrm{Ba}, 199 \mathrm{Hg}, 200 \mathrm{pt}, 80 \mathrm{si}$, 1270 in, $\$ 3$

120 Missourl, Und versity of Colunota, Hissouri 65201

sofe, $122 \mathrm{Te}, 128 \mathrm{Te}$

111 Natlonal bureng oi Stendards Connectlcut Avenue and Van Ness Street, N.W. Washington, D. C. 29234

137ga, socr, s2Cr, s*fe, sofe, $201 \mathrm{Hg}, 13 \mathrm{In}, 2 * \mathrm{Nd}, 123 \mathrm{Sb}, \mathrm{SS}, \mathrm{Tg}$, $30=\mathrm{T} 1$

112 New England Nuclear Corporation 575 Albany Street Boston, Massachusets 02118

$107 \mathrm{Ag}, * \mathrm{Ca}, 11 * \mathrm{Cd}, 50 \mathrm{Cr}, 5+\mathrm{Fe}$, sofe, $1 \mathrm{~K}, 42 \mathrm{NI}, 100 \mathrm{Pd}, 124 \mathrm{Sn}, 126 \mathrm{Te}$ Tgs, 203T1, $100 \mathrm{~W}$

113 New Jargey. College of Medicline and Dentistry 100 Bergen street Newark, Ney Jergey 07103

$207 \mathrm{Ag}$ 
224 New Mexico, Universlty of. Albuquerque. New Mextco 87103

a $R$ b

125 New York Unlversity Medical Center 550 first Avenue

New York. New York 10016

6LI, TLI

116 New York, State UnIverslty of 3435 Main Street Buffalo, Now York 14214

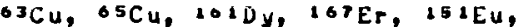
i०5 $\mathrm{Nd}, 1+9 \mathrm{Sm}, 17 \mathrm{iYb}$

117 New York, State University of Research foundation Stony Brook, New York 11790

$106 \mathrm{Cd}, 166 \mathrm{Er}, 50 \mathrm{Fe}, 6 \mathrm{LI}, 145 \mathrm{Nd}$, $150 \mathrm{Nd}, 100 \mathrm{Pd}, 100 \mathrm{Pt}, 106 \mathrm{Pt}, 66 \mathrm{Sr}$, Tyg

118 North Carolina State UnIversity P. D. Box 5935

Raleligh, North Carolina 27607

$+1 \mathrm{~K}, 30 \mathrm{Sl}, \mathrm{SS}, \mathrm{Tgs}$

119 North Carolina, University of Chapel Hill. North Carollna 27514

$3501,5 S, 125 T e$

120 Northeast Utllities Service Company P. 0. Box 270

Hartford. Connecticut 06101

$>\mathrm{Li}$

121 Northern Illinols UnIversity Box 185

Dekalb, Illinolg 60115

$1 \cdot 3 \mathrm{Nd}, \mathrm{SS}, 1 \mathrm{~B}^{3 \mathrm{H}}$

122 Northern States Power Company

414 Nicollet Mal

Minneapolis, Minne sota 55401

$7 \mathrm{Li}$

123 Northwestern University 619 Clark Street

Evanston; Ibllnois 60201

$+\mathrm{OCa}^{+}+{ }^{3 \mathrm{Ca}},+\mathrm{Ca}^{\mathrm{C}}, \mathrm{Tg} 3$

124 Notre Dame, Univer sity of Notre Dame, Indiana 46556

$107 \mathrm{Ay}, 100 \mathrm{Ag}, 110 \mathrm{Cd}, 111 \mathrm{Cd}, 113 \mathrm{Cd}$

$70 \mathrm{Ge}, 72 \mathrm{Ge}, 10 \mathrm{Ge}, 76 \mathrm{Ge}, 208 \mathrm{~Pb}$

$70 \mathrm{Se}, 70 \mathrm{Se}, 60 \mathrm{Se}, 82 \mathrm{Se}, 20 \mathrm{Sl}, \mathrm{Tg}$

125 Dak Ridge Teahnical Enterprlses Corporation 102 HI diand Road

dak Ridge, Tennesses 37830

$\operatorname{Tg} \mathbf{g}$
126 Oberlin College

Cberlin, Ohlo

44074

6L1, JLL

127 Dhio State University

Research foundation

1314 Kinnear Road

Columbus, Ohlo 43212

$11 \mathrm{~B}, 12 \mathrm{C}, 6 \mathrm{~L}$

128 Ohio University

Athens, Ohio. 45701

$6 \mathrm{LI}, \mathrm{TLI}, \mathrm{SSH}_{\mathrm{SS}} \mathrm{Tg}$

129 Oregon State University

Corvallis, Oregon $\$ 7330$

$230 \mathrm{Ba}, 100 \mathrm{Nd}, 109 \mathrm{Sm}, 176 \mathrm{Yb}, 10 \mathrm{Zn}$, $96 \mathrm{Zr}$

130 Oregon, University of Eugene, Uregon 97403

$3 B \mathrm{Ni}, 60 \mathrm{NL}, \mathrm{Tgg}$

131 Pacific Gas and Electric Company P. 0. Hox 7760 San Francisco, Callfornia 94220

7 LI

132 Pacific Northwest Laboratory Richland, washington 99352

$102 \mathrm{Ce}, 168 \mathrm{Nd}, 96 \mathrm{Ru}, 152 \mathrm{~S}, 170 \mathrm{Yb}$

133 Ponngyluania State University University Park, Pennsyluania 16802

Sife

134 Pennsylvania, University of 3025 Walnut street

Phlladelphia, Pennsyluania 19104

79 Ge, $39 \mathrm{~K}, 7 \mathrm{Ll}, 25 \mathrm{Mg}, 110 \mathrm{Pd}, 85 \mathrm{~Kb}$, 10*Ru, IOSe, I>Se, iose, oOSe, $62 \mathrm{Se}, 122 \mathrm{Sn}, 12+\mathrm{Sn}, \mathrm{Tgs}$

135 Pittsburgh, University of PIttsburih, Penngyl vanla 15213

$+2 \mathrm{Ca},{ }^{\circ} \mathrm{Cr}, 162 \mathrm{Er}, 160 \mathrm{Er}, 160 \mathrm{Er}$, $100 E$, $1000 s, 1000 s, 99 R, 100 R u$, $101 R u, 102 R u, 104 R u, 32 S, 23 S I, 30 \mathrm{SI}$, SS, Tys

136 Prinooton IIniueralty James for restal Campus Princeton, New Jersey 08540 63Cu, ${ }^{35 C u}, 54 f e, 6 \mathrm{Ll}, 29 \mathrm{Sl}, 30 \mathrm{Si}$, $70 \mathrm{Zn}$

137 Purdue Un Iversity

Pharoacy Annex Latayetie, Indiaild 4790 ?

I70Er, ISII, TLi, I060g, Tga 
138 Reactor Experiments, Inc.

963 Terminal way

San Carlos, California 94070

$10 \mathrm{~B}$

139 Reed Colleje

$3203 \mathrm{~S}$. E. Hoodstock Boulevard

Portland, Jregon 97202

$76 \mathrm{je}, 76 \mathrm{Se}$

140 Rhode I sland, University of Narrayangett Marine Laboratory KIngston, Rhode Island 02881

$160 \mathrm{Gd}, \mathrm{Igs}_{8 \mathrm{~s}}$

141 Kochester, University of

575 Elmwood Avenue

Rochester, New York 14627

$136 \mathrm{Ba}, 136 \mathrm{Ba}, 138 \mathrm{Ba}, 82 \mathrm{Br}, 200 \mathrm{Ce}$,

$1.2 \mathrm{Ce}, 76 \mathrm{Ge}, 6 \mathrm{LI}, 1+2 \mathrm{Nd}, 1+4 \mathrm{Nd}, 160 \mathrm{Nd}$,

$58 \mathrm{Ni}, 1000 \mathrm{~s}, 128 \mathrm{Os}, 1000 \mathrm{~s}, 1920 \mathrm{~g}$,

$100 \mathrm{Pt}, 02 \mathrm{Se}, 20 \mathrm{Si}, 1+0 \mathrm{Sm}, 1+8 \mathrm{Sm}$.

$128 \mathrm{Te}, 102 \mathrm{~W}, 104 \mathrm{~W}, 106 \mathrm{~W}$

142 Rohw and Haas Company

Independence Mal 1 We

Philadelphla, Pennsyluanla 19105

$>\mathrm{Li}$

143 Rutgers, The State University New Brunswick. New Jergey 08903

$107 \mathrm{Ag}, 130 \mathrm{da}, 132 \mathrm{Ha}, 130 \mathrm{Ba}, 136 \mathrm{Ba}$,

5.Fe, 50Fe, is6Gd, iozpd, iose,

rrse, raSe, sose, $125 e, 125 n$,

lisin, orzn, os $\mathrm{Zn}, 70 \mathrm{Zn}$

144 SAI Technologles

$40 \ddot{n} 0$ Sorronto Valley Duulevard

Sen Diego, Callfornia 92121

$\circ \mathrm{LI}$

145 Sandia Corporation

post uffice box 5800

Albuquerque, Neo Mexico 87115

OLI, 60 MO, 100MO, SONI, GINI,

146 Snlthsonian Institution

10 th Street and Conotitution Avenue N. $H$. Washington, D. C. 20560

Tgs

147 Soutn Carolina, University of

Columbla, South Carolina 29208

* oca, SS, Tgs

148 Southern Calleornia, University of University Park

Los Angeles, Californta 90007

$>9 \mathrm{Br}, \triangle \mathrm{Br}$
149 Southern Hethodist University Purchasing Department

Dallas, Toxas 75275

$120 \mathrm{Cd}, 92 \mathrm{Mo}, 200 \mathrm{~Pb}, 120 \mathrm{Sn}, 66 \mathrm{Sr}$, $130 \mathrm{Te}, 205 \mathrm{Ti}$

150 Spectra-Mat, Inc.

1240 Hlgnuay 1

Hatsonville, California 95076

6LI, ILI

151 Spectra-Physics, Inc.

1250 H. Middlefíld Road

Hountaln View, Call fornie 94040

11*Cd, SS

1 s2 Stanford UnIversity

P. D. Box 4409

Etanford, Callirurila 9450 s

$107 \mathrm{Ag}, 11 \mathrm{~B}, 200 \mathrm{Hg}, 4 \mathrm{~K}, 24 \mathrm{Mg}, 05 \mathrm{Rb}$, $170 Y \mathrm{~b}$

153 Syracuse University

150 Marshall Street

Syracuse, New York 13210

$6>2 n$

154 Teledyne I sotopes

123 Woodland Avenue

Hestwood. New Jarsey 07675

$20 \bullet P b$

155 Tennessee. University of

Knoxullie, Tennessae 37916

Igo

156 Texas $A$ and $M$ University

Post Oefice Box 44

College Station, Texas 77843

*Ca, I53Eu, $150 \mathrm{Gd}, 72 \mathrm{Ge}, \cdot \mathrm{LI}, 150 \mathrm{Nd}$,

$60 N 1,200 \mathrm{~Pb}, 32 \mathrm{~S}, 123 \mathrm{Sb}, 70 \mathrm{~S}$,

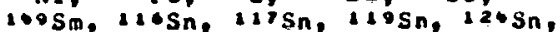

$120 \mathrm{Te}, 130 \mathrm{Te}, 0 \mathrm{Tl}, * 2 \mathrm{Zn}, 0 \mathrm{Zn}$

157 Toledo Edison Caonany and

Cleveland Electric Illueinating Company

5501 North State Route No. 2

Dak Harbor, Ohlo 43449

ILI

158 U. S. ERDA

Morgantoun Energy Research Centur

Collins Ferry Road

Morgantoun, West Virginla 26505

ILI. Tys

159 U. S. Energy Research and Developnent Administration Purchasing orfice 4800 Forbes Avenue

Pittsburgh, Pennsyl vanta 15213

$53 \mathrm{Cr}, 200 \mathrm{~Pb}, \quad{ }^{68 \mathrm{Zn}}$ 
160 U. S. Navy

Director of Supply

Naval Undersea Center

San viego, Calicornia 92132

$10_{B}, O L I, T g S$

161 Union Carbide Corporation

Sterling forest Research Center

post offlce Box 324

Tuxedo, New York 10987

socr, 0osr, SS

162 Union Carblde Corporation, Nuclear Division Cak Kidge National Laboratory

Post Office Box $x$

Dak Ridge, Tennessee 37630

$10 \mathrm{~B}, 1 \mathrm{1B},{ }^{\circ} \mathrm{Ca}, 52 \mathrm{Cr}, 5 \times \mathrm{Cr},{ }^{3} \mathrm{Cu}$,

$153 \mathrm{Eu}, 56 \mathrm{fe}, \mathrm{sife}, 58 \mathrm{fe}, \mathrm{iga}$,

is2Gd, 1soid, $150 \mathrm{gd}, 158 \mathrm{Gd}, 260 \mathrm{Gd}$,

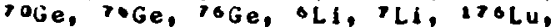

$2+\mathrm{Mg}, 23 \mathrm{Mg}, 26 \mathrm{Mg}, 95 \mathrm{MO}, 1+2 \mathrm{Nd}, 150 \mathrm{Nd}$

$50 \mathrm{NI}, 60 \mathrm{Ni}, 62 \mathrm{Ni}, 207 \mathrm{~Pb}, 200 \mathrm{~Pb}$,

$100 \mathrm{Pd}, 110 \mathrm{Pd}, 195 \mathrm{Pt}, 106 \mathrm{Pt}, 100 \mathrm{Ru}$,

$101 R 4,102 R u, 100 k 4,28 \mathrm{SI}, 100 \mathrm{~S}$,

154 Su, 126 Sn, $1105 \mathrm{~S}, 120 \mathrm{Sn}, \mathrm{SS}, 122 \mathrm{Te}$,

$120 \mathrm{Te}, \mathrm{Tgs}, \bullet \mathrm{Ti}, \cdots \mathrm{TI}, 20 \mathrm{Tl}, 180 \mathrm{~W}$,

$102 W,>0 Z n, 00 \mathrm{Zr}$

163 Union Carbide Corporation, Nuclear Division $Y-12$ Plant

Post Orfice Box $Y$

Dak Hidge, Tennessee 37830

$137 \mathrm{Ba}, 120 \mathrm{Cd}, 65 \mathrm{Cu}, 170 \mathrm{Er}, 100 \mathrm{Hg}$,

$25 \mathrm{HY,} / 50 \mathrm{Nd}, 200 \mathrm{~Pb}, 2 \mathrm{Rb}, 109 \mathrm{Sm}$

- $T$ Ti, $672 n$.

164 United Stateg Army

Ariaed Forces Radioblology Research Inst itute

Defonse Nuclear Agency

Bethesda, Maryland 20014

STFO, Tgs

165 United Stateg Departaent of Agrlculture Research Service

P. 0 . Box 53326

New Urleans, Loulsiana 70150

S*Fe, Sofe

166 United States Department of Interlor Geology Survey

Bldg. 21, Denver Federal Center

Denver, Colorado 80225

- K K, OLI, >LL

167 United States Department of Justice Ninth and Pennsylivania Avenue, N.W. Washington, D. C. 20535

$\bullet \mathrm{Ca},+1 \mathrm{~K}, 26 \mathrm{Hg}, 10 \mathrm{Se}, 60 \mathrm{Zn}$

168 United States Geological Survey

Financial Management

National Center, Room 1447-A

12201 Sunrlse Valley Drive

Reston, Virginia 22092

6LI, LLI, $1890 \mathrm{~g}, 1900 \mathrm{~s}, 65 \mathrm{Hb}$,

$\triangle R$ i IOSRe
169 United States National Aeronautlcs and Space Adm. Lewls Research Center

21000 Brookpark Road

Cleveland, Ohio 44135

157Gd, $160 \mathrm{Gd}, 1600 \mathrm{~g}, 109 \mathrm{Sg}, 110 \mathrm{Sn}$,

Tgs, $203 T$ l, $1,2 \mathrm{~W}$

170 Unlted States National Aeronautics and Space Adm. Goddard Spoce Flight Center Glendale Hood

Greenbelt, Maryland 20771

$100 \mathrm{Hg}, 202 \mathrm{Hg}$

171 United States National Aeronautics and Scace Admo Langley Research Center Langley Station

Hampton, VIrgInia 23365

$102 \mathrm{Pd}, 102 \mathrm{Ru}$

172 Unlted States National Aeronautics and Space Adm. Ames Research Center Morfett Field, Callifornla 94035

$\bullet \mathrm{OCa}, * \mathrm{Ca}$

173 United States Navy

Naval Research Laboratory

Washinjton, D. C. 20390

ISIEU, SS

174 United States Puolic Health Service Nat I onal Institutes of Health, Isotope Laboratory Bullding 21

Bethesda, Maryland 20014

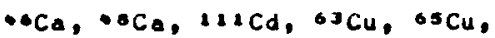

$199 \mathrm{Hg}, 1 \mathrm{Se}, 129 \mathrm{Sn}, 20 \mathrm{Tl}, 70 \mathrm{Zn}$

175 UnIversal Dil Products Company

30 Algonquín Road

Des Plaines, Illinols 60016

oL

176 UnIversity of Connecticut

Heal th Center

Faraington, Connecticut 06032

$106 \mathrm{Cd}, 1 \mathrm{1} 1 \mathrm{Cd}, 110 \mathrm{Cd}$

177 Unt versity of Lowel

Lowell. Massachusetts 01854

$16 \bullet D y$

178 Utah, University of

Purchasing Department

Salt Lake City, Utah 84112

- ${ }^{3} \mathrm{Cu}, * 5 \mathrm{Cu}$

179 Vanderbllt Universh ty

Business Managers office

Nashullie, Tennessee

37203

aBr, 3scl, SS 
180 Various Miscellaneous Blllings Dornestic

$63 \mathrm{Cu},{ }^{\circ} \mathrm{Cu}, \mathrm{Tgs}$

181 Veterans Administration Hospl tal 3801 Junipero Serra BI vd. Palo Alto, California 94304

$\checkmark \mathrm{Ca}$

182 Virginia Electric and Power Company P. O. Box 1194 Richmond, Virginia 23209 ILi

1a3 Virginia Palytechnic Institute Blacksburg. Virginia 24061

$166 \mathrm{Nd}, 1900 \mathrm{~g}, 187 \mathrm{Ke}, 14 \mathrm{SS}, \mathrm{Tgg}$

184 Virginla, University of Charlottesullie, Virginia

$107 \mathrm{Ag}, 100 \mathrm{Ag}, 1 \mathrm{~B}, 113 \mathrm{Cd}, 100 \mathrm{Hg}$, $26 \mathrm{Mg}, 152 \mathrm{Sin}, \mathrm{Tg} S$

185 Washington State University Coinptroller's office

Puliman, Washington 99163

$11 . \mathrm{Cd}$

186 Washington, Univergity of 400 Adoinlstration Bullding Seattle. Washington 98106

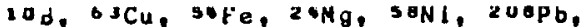
SS, Tys

187 West Virginla University Adainistrotion building Morgantown, Mest Virginia 26506

STFe

188 Western Michigan University

Kal anazoo, Michigan $\$ 9001$

1.2Nd, 144Nd, 146Nd, SS

189 Hestinghouse tilectric Corporation Bettis Atomic Power Laboratory

Post Defice Box 79

Host Aiffilin, Ponnsyluinia 1613 3

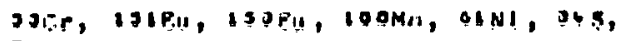
Tgs

190 Hestinghouse Electric Corporation Post Deflce Bax 284

Elmira. New York 14902

$6 \mathrm{Li}$

191 Hestinghouse Electric Corporation Atoric Power Divisions P. D. Box 355

Plttgburgh. Pennsylivania 15230

ILI
192 Westinghouse Hanford Company

P. O. Box 1970

Rlchland, Weshington 99352

Tgs

193 Uisconsin. Uni versity of 750 Uni verslty Avenue Madison, Wi sconsin 53706

$13 * \mathrm{Ba}, * 3 \mathrm{Cu}, 6 \mathrm{Ll}, 20 \mathrm{Hy}, 26 \mathrm{Mg}, 95 \mathrm{MO}$, $.0 \mathrm{HO}, 06 \mathrm{Ru}, 64 \mathrm{Zn}, 60 \mathrm{Zn}, 6 . \mathrm{Zn}$

194 Hoods Hol o Oceanographic Inst itute Woods Hole, Massachugetts 02543

$2951,305 i$

195 Yale Unlversity

20 Ashaun Street

Nev Haven, Connecticut 06520

-2ca, *ca, ilsca, tli; 2451,2051 , $\mathrm{TgS}, \sin , \bullet \mathrm{Zn}$

196 Yeshiva University 1300 Morria Park Avenue Bronx. Naw York 10461

- scu

$$
\text { B . FORE IGN }
$$

ARGENTINA

197 Coalsion Naolonal de Enorgla Atomica Gerencla de Energla

Auda del llbertadó Bटjo

Buenos Alres. Argentina

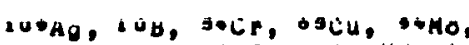

$97 \mathrm{MO}, 1.2 \mathrm{Nd}, 1+3 \mathrm{Nd}, \mathrm{l}+\mathrm{Nd}, 1+5 \mathrm{Nd}$,

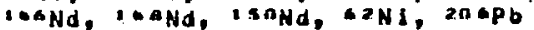

AUSTRALIA

198 Australtan Consulate General 636 Fifth Avenue

New York, New York 10020

$208 \mathrm{~Pb}$

1yy Australlan National University

P. 0. Box 4

Canberra, A. C. T., Australia

$130 \mathrm{Ba}, 13 \mathrm{sBa}, 136 \mathrm{Ba}, 162 \mathrm{Dy}, 260 \mathrm{Er}$

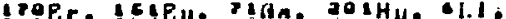

9 SMO, $150 \mathrm{Nd,} 100 \mathrm{Pd}, 106 \mathrm{Pd}, 108 \mathrm{Pd}$,

lopd, IOSe, ose, ose, Josi.

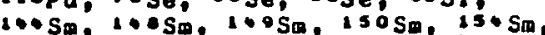

$110 \mathrm{Sn}, 120 \mathrm{Sn}, 122 \mathrm{sn}, \mathrm{SS}, \mathrm{Tgg}$

200 Comonwalth Scientific and Ind. Res. Orgenization Division of Applied Physics Uni versity Grounds, City Hoad, Chippendale N.

Sydney, N. S. H., Australle

23૯La, $17 \bullet L U, Q 7 R b$

201 Mel bourne, Unl versity of Parkille N.2, Melbourne Victoria, Australia

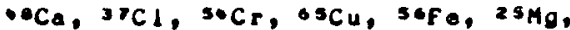

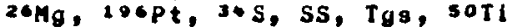


202 Monash Unlversity

Wellington Road

Clayton, Victoria, Australla

$160 \mathrm{Dy}, 100 \mathrm{Er}$

203 Western Australian Institute of Technology Hayman Road

Bentley South 6102

Hestern Australia

$175 \mathrm{Lu}$

\section{AUSTRIA}

204 International Atomlc Energy Agency Kaerntnerring $11-13$

A-1010 Vienna, Austria

$135 \mathrm{Ba}, 136 \mathrm{Ba}, 137 \mathrm{Ba}, 156 \mathrm{Gd}, 15 \mathrm{Gd}$, $150 \mathrm{Gd}, 191 \mathrm{I}, \circ \mathrm{Ll}, \mathrm{TLI}, 24 \mathrm{Mg}, 25 \mathrm{Hg}$, $26 \mathrm{Mg}, 150 \mathrm{Nd}, 1860 \mathrm{OS}, 190 \mathrm{OS}, 100 \mathrm{Pd}$,

Tgs

\section{BELGI UM}

205 Centre d'Etude de l'Energie Nucleal re 144 Avenue Plasky Brussels 4, Belyium

$152 \mathrm{Gd}$

206 European At omic Energy Commission Central Bureau for Nuclear Measurements Geel, Belgium

$50 \mathrm{Cr}, 52 \mathrm{Cr}, 291 \mathrm{Eu}, 5 * \mathrm{Fe}, 56 \mathrm{Fe}$,

STFe, SS, Tgg

207 IRE, Instltut National des Radloelements Avenue Eugene Plasky, 144

1040 Brussels, Belglum

$79 \mathrm{dr}, 82 \mathrm{Br}, 202 \mathrm{Hg}, \mathrm{SS}$

208 Liege, Universite de 2 rue Arwand Stevart Llege, Belglum

92 ,40

209 Louvain, Universite de Parc D'Aremberg

Heverle Louvalin, Belglum

Li, TLl, $140 \mathrm{Nd}, 190 \mathrm{Nd}, 1670 \mathrm{~s}$,

110 d, IS०Sm, I20Te, I20Te, i20Te

BRAZIL

210 Fropesas Nurilearea Rrasilelras S/A - Nuclebrag Rua General Polidoro

316 - Rlo de Janel ro (RJ)

Braz!l

2.2HO, 1*2Nd

211 Governo Federal, Conge tho Nacional de Pesquisas Instituto de Energia Atomica

Calza Fostal 11049

Sao Paulo 9, S. P. A., Brazl

$112 \sin ; 5 S ; \mathrm{Tg} 0$
212 Hospltal das CIInicas da Faculdade de Medicina da Uníversldade de Sao Paulo Sao Paulo, Brazll

$136 \mathrm{Ba}, 1+2 \mathrm{Ce}, 1+3 \mathrm{Nd}$

CANADA

213 Atomlc Energy of Canada Limited

P. D. Box 6300

Postal Station J

Ottawa, Canada

$50 \mathrm{Cr}$

214 Atomic Energy of Canada Linlted Chalk River, Ontario, Canada

$10 \mathrm{~B}, 14 \mathrm{H},+2 \mathrm{Ca}, 121 \mathrm{Cd},{ }^{\circ} \mathrm{Cu},{ }^{\circ} \mathrm{Cu}$, $151 \mathrm{Eu}, 253 \mathrm{Eu}, 76 \mathrm{Ge}, 109 \mathrm{Hg}, 200 \mathrm{Hg}$, $204 \mathrm{Hg}, 1 \mathrm{~K}, \quad \mathrm{LI}, 2+\mathrm{H}_{4}, 5 \mathrm{NI}, 60 \mathrm{Ni}$. $206 \mathrm{~Pb}, 110 \mathrm{Pd}, 96 \mathrm{Ru}, \mathrm{FSE}, \mathrm{TSE}$,

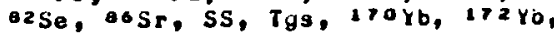
$110 \mathrm{Yb}, 176 \mathrm{Yb}, 90 \mathrm{Zr}, 91 \mathrm{Zr}, 92 \mathrm{Lr}$, $9 \cdot Z \mathbf{r}, 9 \bullet Z \mathbf{r}$

215 Atomic Energy of Canada Límited Whiteshell Nuclear Research Establishoent PInawa, Manitoba, Canada

108

216 British Columbla, University of Vancouver 8, B.C., Canada

$35 \mathrm{Cl}, 208 \mathrm{~Pb}$

217 Dalhousie Univergity Hall fax, Nova Scotia Canada

$101 \mathrm{Dy}, 163 \mathrm{Dy}, 160 \mathrm{Gd}, 1020 \mathrm{~s}, 196 \mathrm{Pt}$, $10 \mathrm{Sn}$

218 Gueloh, Und verslty of Guelph, Ontario, Canada

$102 R u$

219 Health Sclences Centro

791 Notre Dane Avenue Winnipeg, Manltoba, Canada R3E OM 1

$12 \cdot T e$

220 McGill University.

Montreal 2, P. Q., Canada

$130 \mathrm{Ba}, 19 \mathrm{Br}, 4 \mathrm{Br}, 1 * \mathrm{Sa}, 122 \mathrm{Sn}, 12 * \mathrm{Sn}$

$22 i$ McMaster University Hailiton, Ontario, Canada

$10 \mathrm{~B}, 136 \mathrm{Ba}, 1+0 \mathrm{Ce}, 1+2 \mathrm{Ce}, 161 \mathrm{DV}$, $163 \mathrm{Dy}, 160 \mathrm{Dy}, 162 \mathrm{Er}, 160 \mathrm{Er}, 160 \mathrm{Er}$, $200 \mathrm{Er}, 170 \mathrm{Er}, 151 \mathrm{Eu}$, S+FE, $170 \mathrm{HL}$. $102 \mathrm{Nd}, 20 \mathrm{Nd}, 146 \mathrm{Nd}, 16 \mathrm{Nd}, 150 \mathrm{Nd}$, $70 \mathrm{Se}, 7 \mathrm{Se}, 70 \mathrm{Se}, 0 \mathrm{Se}, \mathrm{aSe}$, $105 \mathrm{Sm}, 108 \mathrm{~S}, \mathrm{SS}, \mathrm{Tgg}, 50 \mathrm{Jl}, 172 \mathrm{Yb}^{\circ}$ $176 \mathrm{Yb}, 60 \mathrm{Zn}, 92 \mathrm{Zr}$

2a2 MeNaster Unlversity Modfiol Contor Fursy the Avenue Hadilon, Ontario, Canado

150Dy, $4 L I, ~ 1525 m$ 
223 Merck, Sharp and Dohme

Canada limited

16711 Trang Canada Highway

KIrkland, Quebec, Canada

$12 \mathrm{~B}, 13 \mathrm{Ca}, 25 \mathrm{Mg}, 7 \mathrm{Se}, 125 \mathrm{Te}$

224 Montreal, Univergite de Montreal, Canada

$52 \mathrm{Cr}, 96 \mathrm{Ru}, 90 \mathrm{Ru}, 99 \mathrm{Ru}, 100 \mathrm{Ru}$, $102 \mathrm{Ru}, 10+R u, 7+3 e, S S, I y s,+8 T \mathrm{I}$

225 National Research Counci

Montreal Road Laboratorias

Ottawa, Ontario, Canada

$11 \mathrm{~B}, 61 \mathrm{Br}, 37 \mathrm{Cl},{ }^{3} \mathrm{Cu}, 63 \mathrm{Cu}, 73 \mathrm{Ge}$,

$58 \mathrm{Nl}, 60 \mathrm{NI}, 60 \mathrm{Ni}, 1800 \mathrm{~s}, 1890 \mathrm{~s}$,

$10009,10205,113 n$

22.6 Quaens UnIversity

Klilgston, Ontarlo, Ganada

$-0_{\mathrm{Ca}}, 15 \mathrm{In}, 28 \mathrm{SI}, 117 \mathrm{Sn}, \mathrm{SS}, 92 \mathrm{Zr}$

227 Saskatchewan, Universlty of

Saskat oon, Saskatchewan, Canada

$107 \mathrm{Ag}, 92 \mathrm{M0}, 11 \mathrm{sn}, \mathrm{Tgs}$

228 Scintrex, Ltd.

222 Snldercroft hoad

Concord. Ontario, Canada

$206 \mathrm{Po}, 207 \mathrm{~Pb}, 208 \mathrm{~Pb} ; \mathrm{Tgs}$

229 Simon Fraser University

Burnaby 2 , B. C.. Canada

$123 \mathrm{Te}, 125 \mathrm{Te}$

LsU loronzo, unlverslly uf

Toronto, Ontario, Canada

$152 E U, 24 \mathrm{H}_{\mathrm{G}}, 25 \mathrm{Mg}_{\mathrm{g}}$

231 University of Alberta

Edmonton, Alberta, Canada T6G2HI

OLE, 11K, 64N1, $120 \mathrm{Sn}, 122 \mathrm{Sn}$

237. Inniversity of Calaary

2920-24th Avenue Northwest

Calgary, Alberta, Canada T2N IN4

$160 \mathrm{Gd}$

DENMA RK

233 Aarhus, Univeralty of Aarhus $C$, Denmark

13. Ba

234 At omic Energy Coamision Research Establishment Riso

Iso tope DIvision

Roskilde. Denmark

* ca, *ca
235 Denaark Technical: University of Lyngby, Denark

s. Fe

236 Kobenhavns Uni versitet

Blegdamsuej 17

Kobenhavn 0 , Denmark

$153 E$ U, $145 \mathrm{sm}$

237 Kobenhavns Unl versi tet Ri so Pr.

Roskilde, Denaark

$107 \mathrm{Ag}, 109 \mathrm{Ag}, 130 \mathrm{Ba}, 135 \mathrm{Ba}, 134 \mathrm{Ba}$, - $\mathrm{Ca}^{\circ}, 2 \mathrm{Ca},+\mathrm{Ca}, 106 \mathrm{Cd}, 100 \mathrm{Cd}$, $110 \mathrm{Cd}, 20 \mathrm{Ce}, 1+2 \mathrm{Ce}, 50 \mathrm{Cr}, 161 \mathrm{Dy}$, $166 \mathrm{Er}, 56 \mathrm{Fe}, 154 \mathrm{Gd}, 156 \mathrm{Gd}, 150 \mathrm{Gd}$

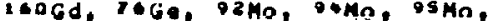
$96 \mathrm{MO}, 97 \mathrm{MO}, 100 \mathrm{HO}, 1+2 \mathrm{Nd}, 163 \mathrm{Nd}$,

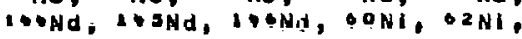
$\triangle N L, 10 \% P d, 106 P d, 100 P d, 120 P d$,

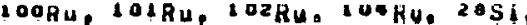
$30 \mathrm{Si}, 1+\mathrm{S}, 1+7 \mathrm{Sa}, 1+9 \mathrm{SE}, 150 \mathrm{~S}$, $120 \mathrm{Sn}, 122 \mathrm{Te}, 120 \mathrm{Te}, 126 \mathrm{Te}, \mathrm{TgS}, 186 \mathrm{Yb}$

\section{ENGLA ND}

238 Eradford. University or Bradford 7, Engl and

søre

239 British Embassy 3100 Massachusetts Avenue, N. W. Washington, D. C. 20008

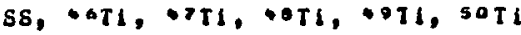

240 Leeds, Uni versity of

Leeds 2, England

$200 \mathrm{Ph}, 200 \mathrm{~Pb}$

241 Levy West Laboratorles, Ltd. Bush Fair Harlow, Essex, Engl and

LI

242 Liverpool, University of l. Iverpuul $3_{\text {, Erivland }}$

$\odot{ }^{\circ} u_{\text {, }} \quad$ Se

243 London, University of Straña, London W.C.2.. Enyland

$2 \sin _{\mathbf{y}}$

244 Manchester University Manchester 13, England

11.B, $137 \mathrm{Ba}, * 0 \mathrm{Ca}, 106 \mathrm{Cd}, 160 \mathrm{Gd}$, 14+Nd, 14\$Nd, t०0NA, 192חs, $20 \circ \mathrm{Pb}$ 90Ru, 1* Se, $107 \mathrm{Sm}, 1+9 \mathrm{Sa}, 132 \mathrm{Sm}$. I s 5 m

245 Oxford, Unl verslty of Nuclear Pnysics Laboratory 21 Banbury Road Oxford, England

Tgs, $\bullet T$ I, soT 
246 R. E. Swann, Ltd.

10y Fenchurch Street

London, E.C.3, England

$102 \mathrm{Ce}, 37 \mathrm{Cl}, 167 \mathrm{Er}, 138 \mathrm{La}, \mathrm{SS}, 171 Y \mathrm{~b}$

247 Radiochemical Centre

White Llon Road

Amersham, Bucks, England

.6Ca, 50C $159 \mathrm{Fa}, 152 \mathrm{Gd}, 190 \mathrm{Hg}$,

SONI, 62NI, "Se, $112 \mathrm{Sn}$, a Sr, SS,

$122 \mathrm{Te}, 203 \mathrm{Tl}, 168 \mathrm{Yb}$

248 St. Mary's Hosplital Medical School

Norfolk Pl. Paddington

London W2 IPG, England

- aca

249 United Kingdom Treasury and Supply Delegation 25 Broadway

Ner York, New York 10004

$10 \mathrm{~B}, 13 \mathrm{rad}, 13 \mathrm{Ba}, \cdots \mathrm{Ca}, 120 \mathrm{Cd}, 110 \mathrm{Cd}$,

$142 \mathrm{Ce}, 37 \mathrm{Cl}, 50 \mathrm{Cr}, 54 \mathrm{Cr}, 162 \mathrm{Dy}$,

$191 \mathrm{Eu}, 70 \mathrm{Ge}, 73 \mathrm{Ge}, 70 \mathrm{Ge}, 179 \mathrm{HP}$,

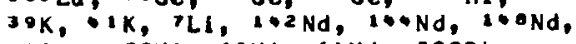

$150 \mathrm{Nd}, \mathrm{SONI}_{\mathrm{N}}, 62 \mathrm{Ni}, 6 \mathrm{NI}, 208 \mathrm{~Pb}$,

$205 i, 29 \mathrm{Si}, 305 i, 145 \mathrm{Sm}, 1+\mathrm{Sa}$,

$150 \mathrm{Sm}, 210 \mathrm{Sn}, 122 \mathrm{Sn}$

FRANCE

250 CEA

Department des Radioelements

B. P. No. 2

91190 Gif Sur Yuet te, France

$100 \mathrm{Ag}$, ${ }^{3} \mathrm{Ca},{ }^{0} \mathrm{Ca}, 52 \mathrm{Cr}, 53 \mathrm{Cr}$,

$160 \mathrm{Er}, 106 \mathrm{Hg}, 204 \mathrm{Hg}, 1 \mathrm{~K}, 139 \mathrm{La}$,

$175 \mathrm{Lu}, 176 \mathrm{Lu}, 26 \mathrm{Mg}, 50 \mathrm{NI}, 60 \mathrm{NI}$,

$1000 s, 100 \mathrm{Pd}, 10 \mathrm{apd}, 190 \mathrm{P} t, 100 \mathrm{Ru}$,

3osi, 154Sg, 12Sn, $116 \mathrm{Sn}, 120 \mathrm{Sn}, \mathrm{a} \times \mathrm{Sr}$,

orsr, o o Sr, SH, 12*Te, $130 T$, T Tg,

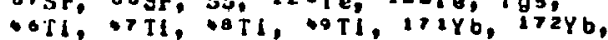

orzn, 902r, 94 $r, 962 r$

251 CRN, Labo PNPA

B. P. $20 / C R O$

67 Strasbourg 3, France

$109 \mathrm{Ag}, 100 \mathrm{Ce}, 162 \mathrm{D} \mathrm{y}, 24 \mathrm{Mg}, 29 \mathrm{Ag}$,

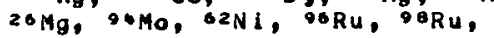

I28Te, ITOYb, Iriyb

252 Grenoble, Univergl te de

Grenoble, France

$12 B, 100 p d, 170 Y b$

253 Institut Max von Laue-Paul Langevin Cedex 156

38 Grenoble-Gare, France

$167 \mathrm{Er}, \bullet 0 \mathrm{~K}, 97 \mathrm{MO}, 1920 \mathrm{~s}, 33 \mathrm{~S}, 12 . \mathrm{Te}$,

$106 \mathrm{~W}, 672 \pi$

254 Institut de Phygique Nuclealre

B. P. No. 1

91 Orgay, France

105 Re
255 Instltut do Physique Nucleaire de Lyon 43 Boulevard du 11 Noveabre 1918 69 Villeurbanne, France

$\mathrm{saCr}_{\mathrm{C}} \mathrm{s}^{3} \mathrm{Cr}, \mathrm{Tgs}$

256 Parls, University of

47 Rue des Ecoles

Paris 5e, France

$92 M_{0}, 100 M_{0}$, Tgs

257 Univerglte de Nantes

Chemin de la Houssiniere

44037 Nantes

France

$3 \times 5$

GERMANY

258 American Hoechst Corporation

P. 0. Box 2500

Somerullie, New Jersey 08876

socr, $190 \mathrm{Hg}, 7+\mathrm{Se}, 04 \mathrm{Sr}, \mathrm{SS}$,

$168 \mathrm{Yb}$

259 Bochum, Ruar-Universitat

463 Bochum, West Geriany

$20 \mathrm{~B}, 3 S \mathrm{CL}, 7 \mathrm{LI}$

260 Bonn, Universitat

Nussalleo 12-14

55 Bonn, Gereany

$11 \mathrm{~B}, 134 \mathrm{Ba}, 135 \mathrm{Ba}, 136 \mathrm{Ba}, 137 \mathrm{Ba}, 192 \mathrm{Pt}$, Tgs

261 Juohl or and Company

Frankfurter Str. 294

Braunschueig, Germany

$10 \mathrm{~B}, * 2 \mathrm{Ca}, * 3 \mathrm{Ca}, * \mathrm{Ca}, *{ }^{\circ} \mathrm{Ca}, 35 \mathrm{Cl}$,

$37 \mathrm{Cl}, 201 \mathrm{Hg}, 26 \mathrm{Hg}, 5 \times N 1,60 \mathrm{Nl}$,

$61 \mathrm{Ni}, 62 \mathrm{NI}, 60 \mathrm{NI}, 20 * \mathrm{~Pb}, 208 \mathrm{~Pb}$,

lospd, 105Pt, 106Pt, 34S, SS,

$68 \mathrm{Zn}$

362 Darmstadt. Technischen Hochschule 61 Darmstadt, Germany

$\bullet 0 \mathrm{Ca}, 39 \mathrm{~K}, * 1 \mathrm{~K}, 1 * \mathrm{Sa}, 150 \mathrm{Sa}, 15 \bullet \mathrm{sm}$,

$\operatorname{Tg} s$

263 Deutsches Elektronen-Synchrotron Desy 2000 Haaburg 52, Notkestleg 1 West Gera any

sife

264 Deutsches Krebgforschungszent rua Derlliner Str. 21

6900 Hel del berg, Germany

$122 \pi 0$

265 Erlangen-Nurnberg, University Gluckstr.

862 Erlangon, Wegt Gereally

$.0 \mathrm{Ca}, 24 \mathrm{Hg}, 20 \mathrm{Mg}, \mathrm{SS}, 602 \mathrm{n}$ 
266 Freiburg/Brsg. Universitat

7800 Freiburg/Brsg.. Germany

$3 \times \mathrm{Cl}, \mathrm{SS}$

267 Frele Universitat

1 Berlin 33, Germany

$168 \mathrm{Er}, 160 \mathrm{Gd}, 200 \mathrm{~Pb}, 112 \mathrm{Sn}, 110 \mathrm{Sn}$,

$117 \mathrm{Sn}, 118 \mathrm{Sn}, 119 \mathrm{Sn}, 125 \mathrm{Te}, 120 \mathrm{Te}$

$130 \mathrm{Te}, \mathrm{TgS}^{2}, 203 \mathrm{TI}$

268 Frele und Hansestadt Hamburg

2 Hamburg-Banrenfeld, Germany

$10 \mathrm{~B}, 150 \mathrm{DY}, 163 \mathrm{DY}, 162 \mathrm{Er}, 100 \mathrm{Er}$,

$151 \mathrm{Eu}, 152 \mathrm{Gd}, 150 \mathrm{Gd}, 160 \mathrm{Gd}, 17 \mathrm{HC}$,

$100 \mathrm{HF}, 101 \mathrm{Ir}, 103 \mathrm{Ir}, 1+3 \mathrm{Nd}, 1+3 \mathrm{Nd}$,

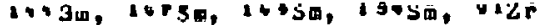

209 تWK-Gesell schati 2 ur Hiederaulbereltuny von Karnb D-705l boopoldshafen, We jt Oefmally

$6 \mathrm{Li}, 20 \mathrm{Mg}$

270 Gesamthochschule Wuppertal

FBY - Prot. Burger

Geverbeschulstr. 34

D-5600 Uuppertal 2

Hest Geroany

$79 \mathrm{Br}, 33 \mathrm{Cl}, 34 \mathrm{~S}$

271 Gesell schaft fur Kernforschung wbH

Institute fuer Angew andte Reaktor Physik

Weberstrasge 5

75 Karlaruhe

West Germany

$10 \mathrm{~B}, 130 \mathrm{Ba}, 132 \mathrm{Ba}, 130 \mathrm{Ba}, 10 \mathrm{Ca}, 110 \mathrm{Cd}$,

$10 \mathrm{Ce}, 50 \mathrm{Fe}, 15 \mathrm{sgd}, 6 \mathrm{Ll}, 26 \mathrm{Mg}$

$200 \mathrm{~Pb}, 0 \mathrm{Sr}, \mathrm{SS}, 120 \mathrm{Te}, \mathrm{Tgs}, 96 \mathrm{Zr}$

272 Gesei i schart fur Schwerionenforschung aBH

Tar ge t Labor

Posteach 541

D-6100 Darmstadt, West Germany

$150 \mathrm{DV}, 20 \mathrm{Mg}, 25 \mathrm{Mg}, 20 \mathrm{Mg}, 00 \mathrm{NI}$,
$206 \mathrm{~Pb}, 207 \mathrm{~Pb}, 208 \mathrm{~Pb}, 108 \mathrm{Pd}, 190 \mathrm{Pt}$, SS, $\mathrm{Tg}, 90 \mathrm{Zr}$

273 Glessen, Universit at

6300 Glogsen. Geraany

$20 * \mathrm{Hg}, 6 \mathrm{LI}, \mathrm{Tli}, 30 \mathrm{SI}, \mathrm{SS}, 182 \mathrm{H}$,

$10 \cdot \mathrm{H}, 106 \mathrm{~W}$

274 Hahn-Mel tner-Inst I tut

Cur Kernforschung Berlin, Sekton Kernphysick Berlla, Germany

$100 \mathrm{HC}, 199 \mathrm{Hg}, 200 \mathrm{Hg}, 103 \mathrm{Nd}, 20 * \mathrm{~Pb}$, $200 \mathrm{~Pb}, 2 \mathrm{a} \mathrm{Pb}, 200 \mathrm{~Pb}, 100 \mathrm{Pd}, 120 \mathrm{Pd}$, a RB, Q12r

275 Heidel berg. Universitat Hel del berg. Germany

$0 \mathrm{Li}, 200 \mathrm{~Pb}, 110 \mathrm{Pd}, \mathrm{Tgg}$

276 Institut rur Elekt rowerkstoffe

Eckerstrasse 4

78 Frelburg, West Germany

$11 \mathrm{~B}, 73 \mathrm{Ge}, 6 \mathrm{NI}, 33 \mathrm{~S}, 29 \mathrm{~S} 1,119 \mathrm{Sn}$
277 Isotopen-Laborator ium

Harfegtr. 148

Cussel dor $t-E l l e r$

Wast Gerany

$10 B$

278 Johann Holfgang Goethe UnIversity Frankfurt/Maln, Germany

5TFe, $150 \mathrm{Gd}, 70 \mathrm{Ge}, 7 \mathrm{LI}, 20 \mathrm{~Pb}$, io०Pt, $190 \mathrm{Pt}, 152 \mathrm{Sm}, \mathrm{Tgs}, \bullet \mathrm{Zn}$

279 Karlsruhe, der Universitat

Engesserstrasse?

75 Karlsruhe. West Geraany

- 3 ca

280 Kernforschung Sanlage

Postlach 365

sit Jullch, Geraany

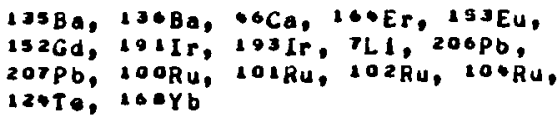

281 Klel Universitat

2057 Goesthacht Reactorstation

$23 \mathrm{Kiel}$, Germany

$106 \mathrm{Cd}$

282 Koln, Uni versitat

Zulpicher str. 47

5 kodn d, Gormany

$100 \mathrm{Cd}, \mathrm{E}+\mathrm{Ag}, \mathrm{CHg}, 2+\mathrm{Hg}, \mathrm{Tgs}$

283 Kriatall handel Kelp In

IHSchlll Ing 16

6906 Lolmon boi Hoidel berg, Hest Gerany

$160 \mathrm{Gd}$

284 List Electrondc

116 Bi soarckstragas

D-61 Darastadt, Wust Geraany

$10+\mathrm{Pt}, \mathcal{I R b}$

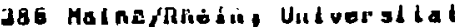

Malnz ae Rhelin, Germany

looch, ABgd, 113Gd; Tgo, 9azr

286 Marburg/Lahn, Unlversitat

Marbury/Lahn, Germany

$20 z R u, 10 * R_{u}, 20 S 1, T g s$

207 Max Planck Instltut fur Kernphysik Sauptercheckweg

Heldelbery, Germany

- $\mathrm{Ca}, 11$ Cd, s*fe, $30 \mathrm{Fe}, 70 \mathrm{Ge}, 76 \mathrm{Ge}$,

$24 \mathrm{Mg}, 23 \mathrm{Mg}, 5 \bullet N i, \cdots N i, 120 \mathrm{Pd}, 122 \mathrm{Sb}$,

$223 \mathrm{Sb}, 14 \mathrm{Sa}, 1+\mathrm{Sa}, \mathrm{s} 2 \mathrm{Sa}, \mathrm{SS}, 120 \mathrm{Te}$

$122 \mathrm{Te}, 120 \mathrm{Te}, 124 \mathrm{Te}, 120 \mathrm{Te}, 130 \mathrm{Ta}, \mathrm{TgB}$

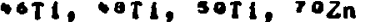


288 Max Planck Inst t tute fur Chemle Saarstrasse 23, Malinz

Hest Geraany

$135 \mathrm{Ba}, 179 \mathrm{HP}, 207 \mathrm{~Pb}$

289 Max-Planck Institut

Bottingerstrasse $6 / 8$,

D34 GottIngull, West Gormany

OLI

290 Munchen, Technischen Hochschule Arcistragse 21

8 Munchen 2

Germany

$10 \mathrm{~B}, 8 \mathrm{Ca}, 102 \mathrm{Ce}, 37 \mathrm{Cl}, 52 \mathrm{Cr}, 160 \mathrm{Dy}$,

$10 \times$ Er, $108 \mathrm{Er}, 150 \mathrm{Gd}$, $150 \mathrm{Gd}$; $160 \mathrm{Gd}$,

T2le, T0Ge, $176 \mathrm{HP}$, BLI, $97 \mathrm{MO}$,

$150 \mathrm{Nd}, 200 \mathrm{~Pb}, 105 \mathrm{Pd}, 106 \mathrm{Pd}, 120 \mathrm{Pd}$,

$192 \mathrm{Pt}, 190 \mathrm{Pt}, 195 \mathrm{Pt}, 106 \mathrm{Pt}, 99 \mathrm{Ru}$,

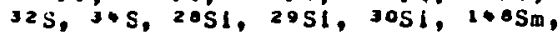

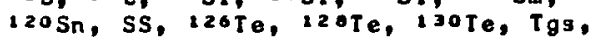

$205 \mathrm{Tl}, 100 \mathrm{~W}, 182 \mathrm{~W}, 180 \mathrm{~W}, 106 \mathrm{~W}$,

$17 a y b, 172 y b, 602 n, 602 n, 902 r$,

$012 r, 922 r, 9<2 r$

291 Munster, Universitat

44 Munster/westialin, Germany

$206 \mathrm{~Pb}$

292 Rohstoft-Einfuhr GmbH

Faunastrasse 61

4000 Duesseldort 1, West Geraany

$196 \mathrm{Hg}$

293 Stuttgort, der Universitat

Azonbargatrasse 12 .

Stuttgart $N$, West Geraany

and, sos 1, Tos

294 Universltat Bielefeld

fakultat fur Physik

Uni varsitatsgtragse

4800 Blelefeld 1

Heat Gieraany

oLi

295 Universltat Konstanz an Glesberg Warenannehme

D-7750 Konstanc

West Gerany

${ }_{63 \mathrm{Cu}}$

296 Universltat Reyens burg

Univargit intsatr. 31

D- 8400 Regensburg

West Germany

$3 \mathrm{Cl}$

INDIA

297 Hhabha Atomic Research Centre

Government of India

3rd Floor Mohat ta Bullding

Boinbay 1, Indi a

$57 \mathrm{Fe}, 200 \mathrm{~Pb}, 04 \mathrm{Sr}, \mathrm{Tg}$
298 Tata Ingti tute of fundamental Research Hol lday Caap, Colaba

Bombay 5, India

B1Br, $70 \mathrm{Ge}, 72 \mathrm{Ge}, 70 \mathrm{Ge}, 93 \mathrm{Mo}$,

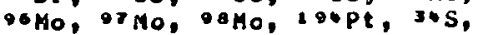

rose, rose, $125 \mathrm{Te}, 64 \mathrm{Zn}, 60 \mathrm{Zn}$

I SRAEL

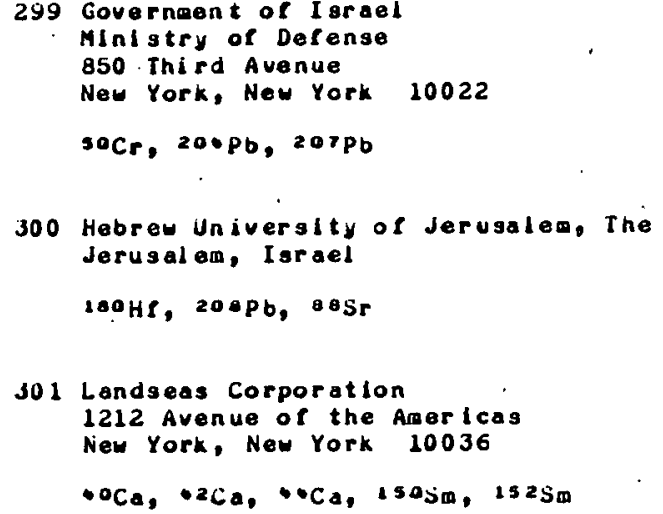

ITALY

302 Comlto Nazlonal 1 Energlo Nucleaire 818 Elghteenth Street, N. H. Washington. D. C. $2000 \mathrm{~s}$

OSMO, SS

303 Istituto Nazionale di Fisica Nucleare Largo E. Fermi 2 50125 Florence, Italy

Tgs, * TI

304 Isti tuto Nazionale di fIsica Nucleare Via A. Tari, 3 80138 Napoli. Italy

-2NI, 28S

305 Ist ituto di Chimlea Biologica Farilita di Medicina E. Chirurgla Universita di Roma 00185 Roma, Italy

- $3 \mathrm{Cu}$

306 Padova, Universita Jeglt Studl di Via F. Marzolo 8

Padova, Italy

$112 \mathrm{Cd}, \mathrm{LI}, \mathrm{PLI}, 30 \mathrm{~S}, 30 \mathrm{~s}, \mathrm{SS}$

307 Univeralty Degi Studi DI Firenze Instituto DI Chemlca farmaceutica VIa 50121 GIno Capponi $S$ florence, Italy

$\because \mathrm{BCu}_{\mathrm{u}}$ 
JAPAN

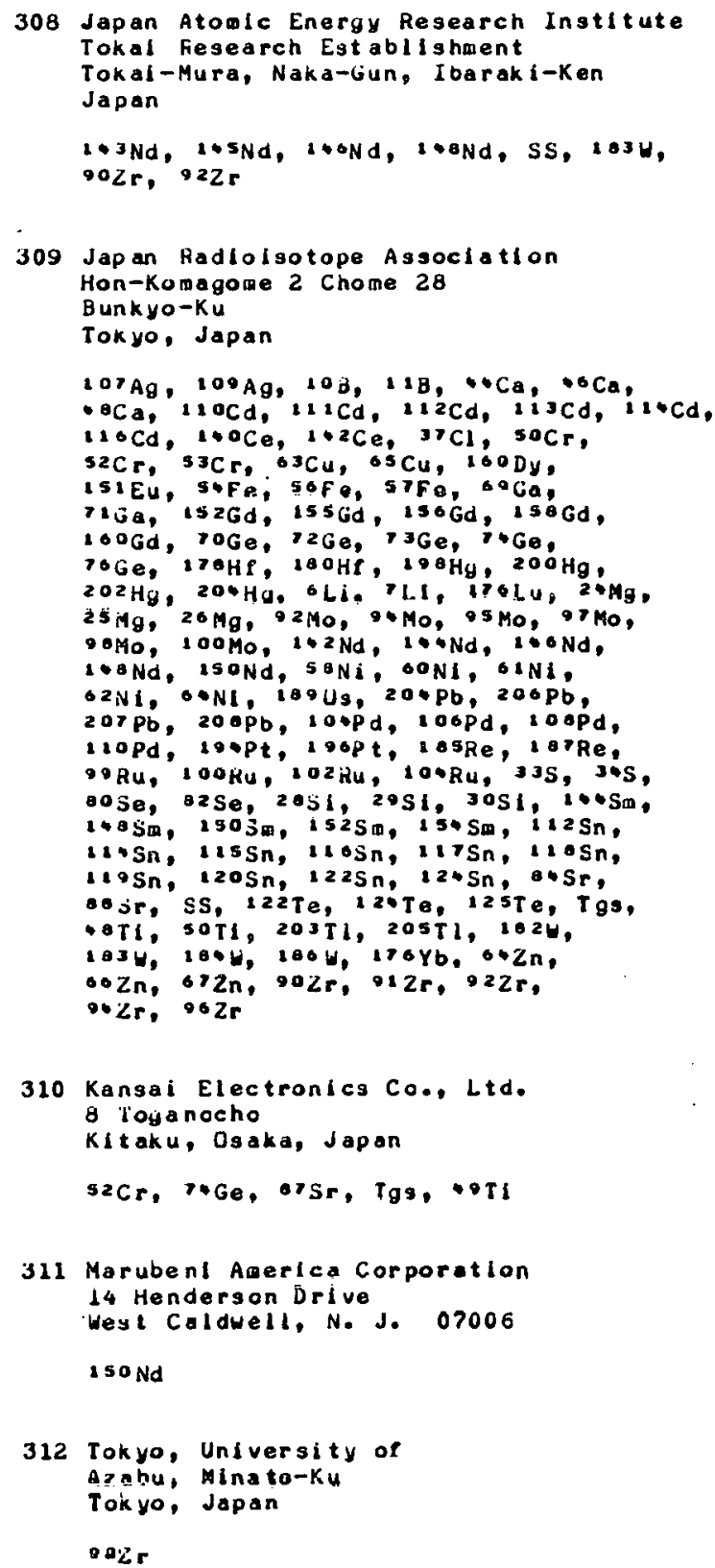

KOREA

313 Kursa Atowic Energy Research Ingtltute P. O. Box 7, Cheong Ryang Seoul, Korea

OLI

\section{MEXICO}

314 U. N. A. M. Instituto de Geofisica Apdo. Pogtal 20-111 Mexico 20 , D. I

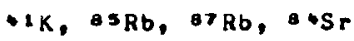

NETHERLANDS

315 Highways International Amsterdamse Straatwey 20 Baarn 2670

The Netherl onds

194pt, 106pt, .0Sr, Tgs

316 Kamerlingh Onnes Laboratorium

Nieuwstegg 18

Lelden

The Netherlands

Is C

317 N. V. Philips-Duphar P. 0. Box 2 Weesp. The Netherlands

20371

318 Reactor Centrue Nederland Petten N. H. The Netherlands

$130 \mathrm{Ce}, 45 \mathrm{Cu}, 150 \mathrm{DY}, 102 \mathrm{E}$, sofe, $71 \mathrm{Ga}, 150 \mathrm{Gd}, 1 \% \mathrm{HC}, 9 \mathrm{MO}^{\circ}, 0 \mathrm{MO}$

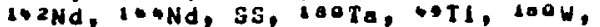
$17 \bullet Y_{b}, 00 \mathrm{Zr}$

319 RIjksinkoopbureau

(Netherlands Govt. Purchasing Of(ice)

S5 A Laan van Heerdervoort

The Hague, Netherlands

$130 \mathrm{Ba}, \bullet 2 \mathrm{Ca}, 3 S \mathrm{Cl}, 14 \mathrm{Er}, 100 \mathrm{EF}$,

STFe, $100 \mathrm{Gd}, 6 \mathrm{LI}, 25 \mathrm{Mg}, 50 \mathrm{NI}$,

$61 \mathrm{Nl}, 200 \mathrm{~Pb}, 100 \mathrm{Pd}, 150 \mathrm{Ba}, \mathrm{SS}$,

$120 T \mathrm{~T}, 126 \mathrm{Te}, \mathrm{TgS}, 140 \mathrm{Yb}, 176 \mathrm{Yb}$

320 State Universlty of Gruningen westersingol 34

ironingen

The Netharlando

$9 \pi u$

321 Vrije Universitelt

Amsterdan, The Netherlands

$130 \mathrm{Ba}, 20 * P \mathrm{~b}, 20+\mathrm{Pd}, 196 \mathrm{Pt}$

NEW ZEALAND

322 University of Auckl and

Private Bag

Auckland, New Zeaband

$+2 \mathrm{Ca}$

NOKWAY

323 Institutt for Atomenergi Kjeller, Norway

-oca, yo Mo

HEPUBLIC OF CONGO

324 Herlot-Watt Uni versity

Riccarton, Edinburgh EH 14 AAS Scot land

$T g s, 1004,02 Z r, 94 Z r$ 
SCOTLAND

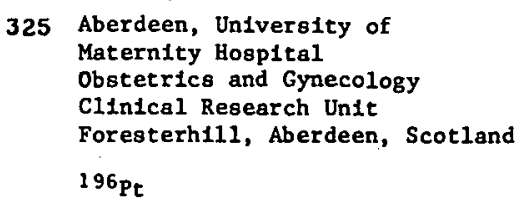

SOUTH AFRICA

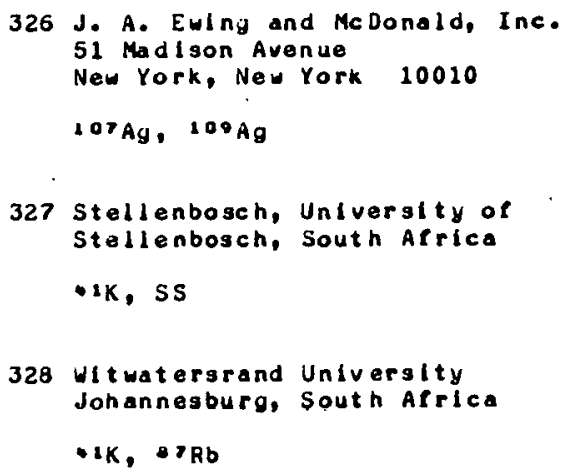

329 Chalmers Univorsity of Technology Gibral targatan $5-b$ Gothenburg, Swaden

${ }^{3} \mathrm{Cu}, \mathrm{TLI}$

330 rorokningelnotitutat Por Atomefik Stockholim 50, Sweden

$102 \mathrm{Nd}, 1 \cdots+\mathrm{Nd}, 1 \cdots \mathrm{Nd}, 132 \mathrm{~T}$

331 The Suedish Ingtitute for Surface Chealstry Deattning Krigtinas Vag 45, s-114 28 Stockholm, Sweden

STFe, SS

332 Universlty of Lund

Sol vegatan 14

Lund, Sweden

$S-22362$

$+3 \mathrm{Ca}, 123 \mathrm{Cd}, 25 \mathrm{Mg}$

333 University of Umea $S-701$ \&7 Umea Sweden

$21+C d$

334 Uppsala, University of Uppsala, Sweden

$100 \mathrm{Cd}, 100 \mathrm{Cd}, 121 \mathrm{Cd}, 112 \mathrm{Cd}, 110 \mathrm{Cd}$, $1.16 \mathrm{Cd},{ }^{3} \mathrm{Cu}$, os Cu, S4Fe, sefe, $142 \mathrm{Nd}, 10 \mathrm{Nd}, 10 \mathrm{Nd}, 150 \mathrm{Nd}$, oinI,

¿2Ni, BONI, YoHU, TOSE, TOKE,

cose, 1505 m
SHITZERLAND

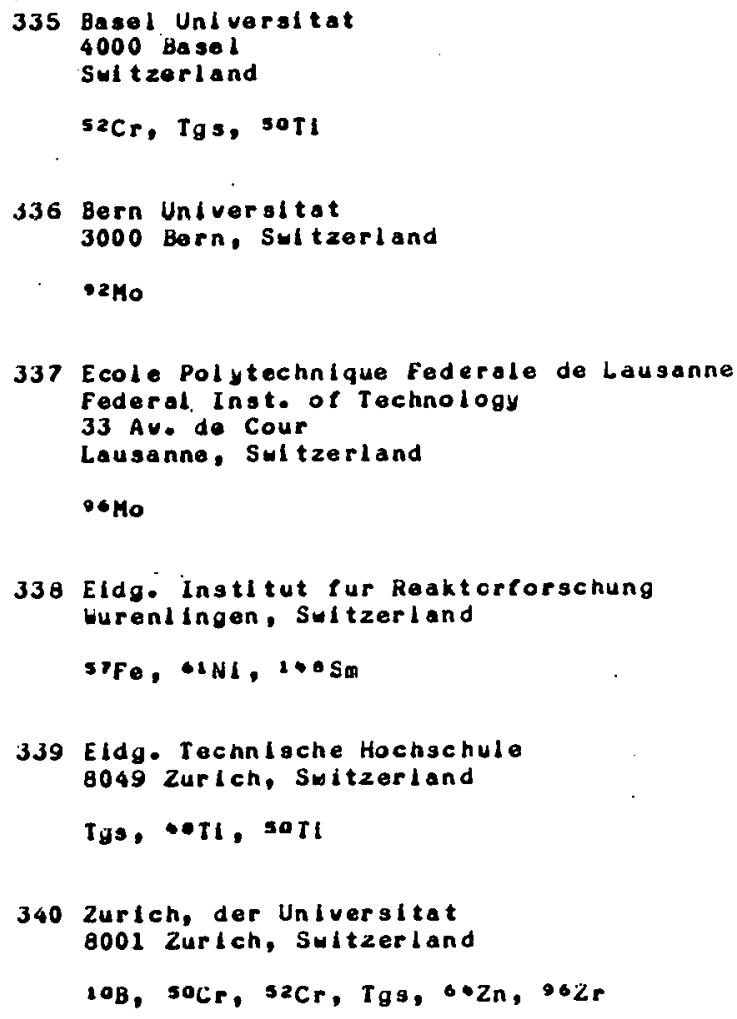

341 Turkey Atomic Eneryy Comalssion Cekmece Nuclear Rosearch Center P.K. - I Hava Alani listanoul. Turkey

SOC $\mathrm{C}$, SOFE, 150Gd, SB, $130 \mathrm{Te}$ 
11. ALPHABETICAL LIST OF ISOTOPES, CROSS-REFERENCED TO CUSTOMER NUMEEHS

A. DOMESTIC

Antiony 123

111, 156

Barl um 130

$35,81,129,143$

Barium 132

$$
67,143
$$

Barlum 134

$$
109,141,143,193
$$

Barlum 135

$$
11,28,49,75,99
$$

Bari um 136

$$
16,109,141,143
$$

Baríum 137

$$
10,99,111,163
$$

Barium 138

$$
3,99,141
$$

Boron 010

$3,18,21,23,34,50,56,59,68,78,84$, $86,138,160,162, .186$

Boron 011

$10,13,28,35,58,127,152,162,184$

Broml ne $07 y$

$8,18,148$

Bromín ne 081

$8,16,64,141,148,179$

Cadm I um 106

$55,117,176$

Cadol Ium 108

84

Cadal um 110

$71,97,124$

Cadal un 111

$47,60,79,124,174,176$

Cadmi um 112

102

Cadmi um 113

$26,32,101,124,184,185,195$

Cadmi um 114

$101,112,149,151,176$

Cadal un 116

21,163
Calclum 040

$10,29,34,36,38,64,84,94,123,162$, 172

Calcium 042

$22,29,42,53,100,135,195$

Calel un 043

49, 123

Calclum 044

$10,27,36,47,64,112,123,156,167,195$

Calc I um 046

$32,36,174$

Calclua 048

$10,24,27,32,82,98,147,172,174,181$

Carbon 012

127

Cariua 136

3

Cerlua 140

35, 141

Cerlua 142

$11,35,64,132,141$

Cnlorlne 035

$32,40,47,82,108,119,17 y$

Chlorine 037

106

Chrodium 050

$36,53,67,111,112,135,161$

Chromium 052

21, jo, 111, 162

Chrom I um 053

53, 159,189

Cnroml un 054

24,162

Copper 063

$10,30,36,40,41,43,93,97,101,116$,

$136,162,174,178,180,186,193$

Copper 065

$10,11,30,36,93,106,116,136,163,174$, $178,180,196$

Dysprosi um 156

11

Dysprosi um 160

10 
Dysprosium 161

$11,64,116$

Dysprosium 164

177

Erbium 162

135

Erbium 164

135

Erbilum 166

$21,40,117,135$

Erblum 167

$11,64,99,116$

Erbium 168

135

Erbium 170

$11,21,35,137,163$

Europ I um 151

$64,81,116,173,189$

Europ I um 153

$11,156,162,189$

Gadol In I um 152

$3,24,162$

Gadol ini un 154

36,166

Gadolinium 155

$9,11,64$

Gadol in ium 156

143,162

Gadol InI um 157

169

Gadolini um 158

21,162

Gadolinlum 160

$10,21,140,162,169$

Gadoliniug High Purity 154

162

Gall I un 069

21

Galll um 071

162

Germani um 070

$124,134,162$
Germanium 072

$$
21,41,124,156
$$

Germanium 074

$$
21,46,124,162
$$

Germani um 076

$124,139,141,162$

Hafni um 178

64

Hafnlue 179

11, 70

Halni un 180

71

Ind ua 113

47,121

Ind lue 125

97, 99

Iridl un 191

36,137

Iridi um 193

11,36

Iron 054

$1,10,35,47,49,53,82,84,88,93,111$, $112,136,143,165,186$

Iron 056

$24,30,35,39,50,84,50,92,162$

Iron 057

$5,18,41,62,66,87,89,106,133,162$, 164,187

Iron 058

$18,36,52,67,99,110,111,112,117,143$, 162,165

Lanthanum $13 y$

3, 11

Lead 204

$16,36,40,47,154,163$

Lead 206

156. 159

Load 207

60,162

Laad 208

$10,21,26,33,34,36,47,84,97,107$, $108,109,124,149,162,186$

LIthl um 006

$3,5,10,17,21,22,34,36,37,39,45$, $50,58,74,84,86,94,55,97,125,117$, $126,127,128,136,141,144,145,150,156$, $158,160,162,166,168,175,190,193,195$ 
LI thi um 007

$4,7,10,12,13,15,16,17,27,33,36$, $37,45,51,59,63,73,74,85,115,120$, $122,126,128,131,134,137,142,150,157$, $162,166,168,182,191$

Lutetiun 175

36,99

Lutet Ium 176

$11,64,162$

Magnesiun 024

$10,24,35,47,64,91,99,107,152,162$, 186,193

Mayriesium 025

$10,13,21,22,24,35,50,55,64,83$, 162,163

Magnesium 026

$10,24,35,49,62,64,84,99,162,167$, 184,153

Mercury 198

$47,77,16.3$

Mercury 199

$31,105,170,174,184$

Mercury 200

47,152

Mercury 201

41,111

Mercury 202

$4 T ; 1 T_{0}$

Mercury 204

$47,77,88$

Molybdenuw 092

$21,36,82,149$

molybdoncim uUb

$10,34,58,162,193$

Molybdenum 096

145,193

Mol yodeñum uy?

10,49

Molyódenum 098

34,47

Malybdenum 100

$38,64,145,189$

Neodymilua 142

$21,28,105,141,162,188$
Neodyolum 143

99, 121

Neodymlum 144

$21,40,105,111,141,188$

Neodyalum 145

$3,11,41,64,59,116,117$

Neodyoium 146

$9,21,36,38,92,141,183,188$

Neodyolum 148

$21,35,41,99,129,132$

Neodyalun 150

$9,21,40,57,70,117,156,162,163$

NIEKeI OSO

$10,35,47,84,92,99,130,141,145,162$, 186

Nickel 060

$10,22,36,84,130,156,162$

Nickel 061

$36,60,145,189$

Nickel 062

$10,19,24,36,53,58,84,112,145,162$ Nickel 064

21, 30, Jo

Osmium 186

35, 50, प्रद, 13\%, 14l,

Osal un 18 ?

35

Osolua 188

$35,135,141$

Osalug 189

168,169

Usmi un 190

$11,24,99,135,141,168,183$

Dam I ua 103

$10,21,36,141$

Palladiue 202

10, $24,143,171$

Palladium 104

10

Palladium 105

21

Palladium 106

$10,21,35$ 
Palladium 108

$10,21,24,38,70,112,117,162$

Palladium 110

$24,41,70,234,262$

Platinum 192

36

PI at Inum 194

$36,47,117$

Plat inum 195

$36.60,162$

PI atinum 196

$18,21,36,41,47,117,141,162$

Potagsium 039

$10,22,33,36,84,99,105,134$

Potassium 040

36,57

Potassium 041

$33,34,36,84,112,118,152,166,267$

RhenI um 185

168

Rhen I um 187

183

Rubldium 085

$10,34,35,36,61,84,134,152,168$

Rubldiug 087

$20,25,32,33,34,61,69,77,84,114$,
163,168

Ruthenium 096

$21,132,193$

Ruthen lum 099

80,135

Ruthenium 100

135,162

Ruthen I um 101

3b, 135,162

Ruthen I um 102

$135,162,171$

Ruthenlua 104

$36,134,135,162$

Samarium 144

$21,35,38,97,99,141,162$

Samar Ium 147

183
Samarium 148

$34,92,141$

Samar fum 149

$11,64,99,116,129,156,163,169$

Samerl un 150

$21,34,42,97,107$

Samar I um 152

$11,21,34,35,92,97,107,132,184$

Samarl um 154

$21,35,38,97,162$

Selenlum 074

96

Salentum 076

$21,124,134,139,143$

Selenium 077

$82,134,143,174$

Selentum 078

$21,124,234,143,156,167$

Selentum 080

$10,47,105,124,134,143$

Selentum 082

34, 105, 124, 134, 141, 143

Silicon 028

$21,36,64,97,124,135,162,195$

silleon 029

$22,84,105,136,141,194,195$

SI $11 \operatorname{con} 030$

$6,22,04,99,105,109,118,135,136,194$ Sllver 107

$10,11,67,112,113,124,143,152,184$

Silver 109

124, 184

Speclal Services

$3,10,16,18,21,27,29,34,35,36,38$, $40,47,50,62,67,75,72,82,84,92,97$, $98,100,105,107,108,109,111,118,119$, 121, 128, 135, 147, $151,161,162,173,179$,

Strontiun 084

$20,36,34,104$

Strontium 086

$10,28,36,47,117,149,161$

strontlum 067

36, 101 
Strontl um 088

35,128

Sulfur 032

$82,135,156$

Sulfur 033

$64,82,105$

sulfur 034

$82,105,189$

Target Preparation stable

$10,14,18,21,24,29,34,35,36,47,48$, $54,58,65,72,44,91,92,94,99,100$,

$107,108,111,112,117,116,123,124,125$,

$123,130,134,135,137,140,146,147,155$,

$158,160,162,164,169,180,183,184,185$, 180, 102, 106

Tellurium 122

$47,102,103,110,162$

Tellurium 124

$30,44,47,102$

Tellurium 125

$$
36,41,44,47,119
$$

Tellurium 126

$$
44,112,162
$$

Tellurium 128

$$
21,41,76,110,141,156
$$

Tellurium 130

$$
31,11,47,110,156
$$

Thall tum 203

$$
27,36,47,111,112,169
$$

That II um 205

$$
10,27,36,47,149,162,174
$$

Tin 112

$$
36,143
$$

$\operatorname{IIn} 114$

$$
21,143
$$

Tin 116

$$
10,36,38,92,156,162
$$

TIn 117

$$
49,109,156
$$

$T \ln 218$

$$
105,108,162
$$

Iin 119

$$
10,33,106,256,169,174
$$

I In 120

$107,108,149,162$
$\operatorname{Iin} 122$

106,134

$\operatorname{Tin} 124$

$21,34,36,106,112,134,156$

Titanium 046

$21,34,58$

Titanlum 047

$44,53,64$

Titanium 040

$18,30,36,38,44,156,162$

Titaniua 048

$24,36,44,64,108,162,163$

Titanium 050

$34,36,49,53$

Tungst en 180

$18,24,76,112,162$

Tungsten 282

$3,48,99,141,162,169$

Tungsten :83

$$
2,3,121,195
$$

Tungs ten 184

$36,99,141$

Tungsten 186

$$
84,141
$$

Yttopblyo 168

$$
24,36
$$

Ytterblum 170

$$
11,99,152
$$

Ytterblua 171

$$
3,11,64,116
$$

Ytterblum 172

$$
10,99
$$

vilertuin 170

$$
3,70,0
$$

Ytterbium 174

$$
38,99
$$

Ytterbium 176

$$
35,129,132
$$

2 inc 064

$$
36,58,64,108,156,153
$$

Zine 066

$58,64,76,106,156,167,193$ 
ZI ne 067

$\frac{11,21,57,82,101,106,108,143,153,}{195}$

Zinc 068

$24,62,64,102,103,143,159,193$

Zinc 070

$36,125,136,143,162,174$

Zireonlum 090

$10,21,36,65,97,162$

21 rconlum osi

$10,11,36$

Zirconlum 092

$21,10.7$

Zirconium 096

$36,47,129$

\section{B. FOREIGN}

Antínony 121

287

Antimony 123

287.

Barium 130

220,271

Barium 132

271

Bariun 134

$199,233,237,260,319$

Barium 135

$199,204,237,260,280,288$

Barium 136

$204,212,221,237,260,271,280,321$

Barium 137

$204,244,249,260$

Barium 138

199,249

Boron 010

197, 214, 215, 221, 249, 259, 261, 268, 271, $277,250,309,340$

Boron 011

$214,223,225,244,252,260,276,309$

Bromi ne 079

$207,220,270$
Bromine 081

$207,220,225,231,248$

Cadol um 106

$237,244,281,282,285,334$

Cadel um 108

$$
237,334
$$

Cadmina 120

$237,271,309$

Cadmi um 111

$214,285,309,334$

CadmI um 112

306, 309, 334

Cadml um 113

$285,30 y, 332$

Cadoium 114

$249,287,309,333,334$

Cadmi um 116

$249,309,334$

Calcium 040

$226,237,262,265,301$

Calctum 042

214, 237, 261,301,319,322

CalcI U⿴囗十 043

$223,250,261,279,332$

Calclum 044

$234,249,261,301,309$

Calcium 046

$234,237,247,280,309$

Calclum 0.8

$201,244,248,250,261,271,287,290,309$, 323

Carlum 138

318

Cerlux 140

221, 237, 201, 271, 300

Ceriua 142

$212,221,237,246,249,290,309$

Cinlorine 035

216, 259, 261, 270,316, 319

Chlorlne 037

201, 225, 246, 249, 261,266,250,296, 309

Chroul um 050

$206,213,247,249,255,258,299,309,340$, 
Chrom lum 052

$206,224,250,290,309,310,335,340$

Chromium 053

$250,255,309$

Chromiura 054

$197,201,237,249$

Copper 063

$214,225,295,305,307,309,329,334$ Copper 065

$197,201,214,225,309,318,334$

Dysprusium $15 \theta$

272

Dyopegium 158

222。 268,318

Dysprosi um 160

202. 290,309

Dysprosium 161

$217,221,237$

Dysprosium 162

$199,249,251$

Dysprosium 163

$217,221,268$

Dysprosium 164

221

E. LI UII 102

$221,2 \epsilon 8,318$

Erbium 164

$221,280,290,319$

Erbium 166

$202,221,237$

Erblum 167

246,253

Erbl um 168

$19 y, 221,260,267,268,290,319$

Erbium 170

199,221

Europium 151

$199,206,214,221,230,249,268,309$

Europium 153

$214,236,280$

Gadol ini um 152

$205,247,268,280,309$

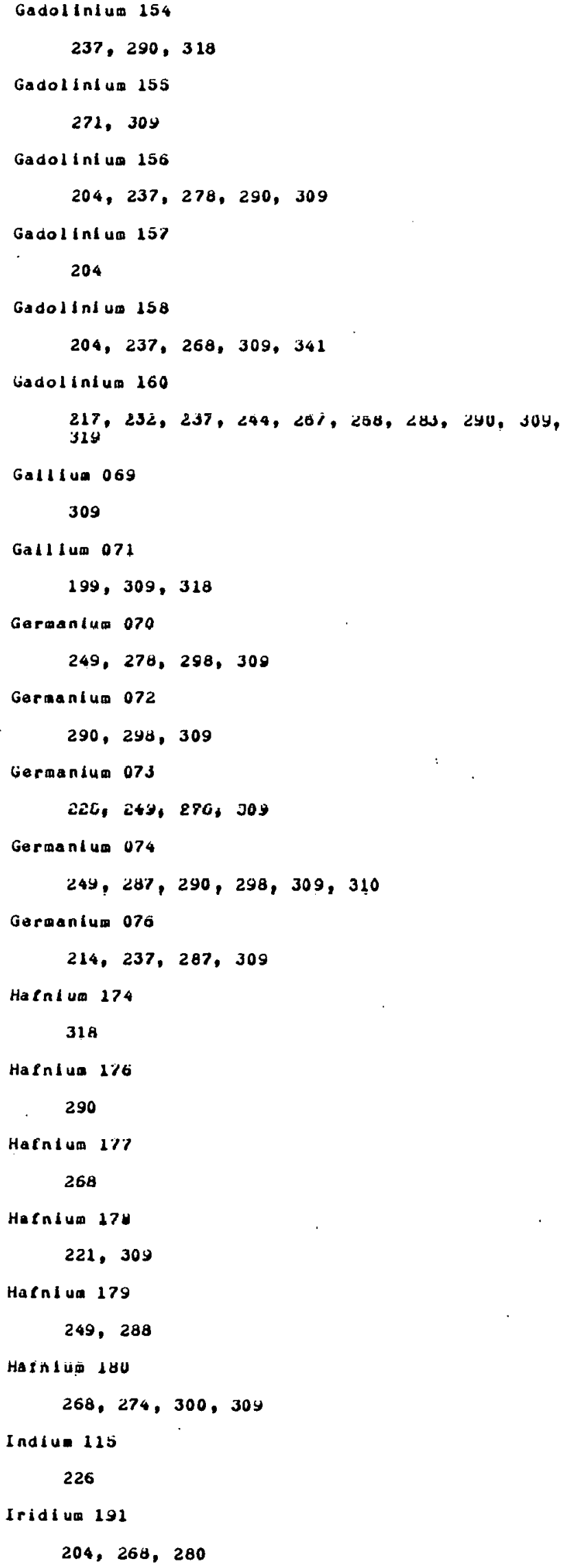




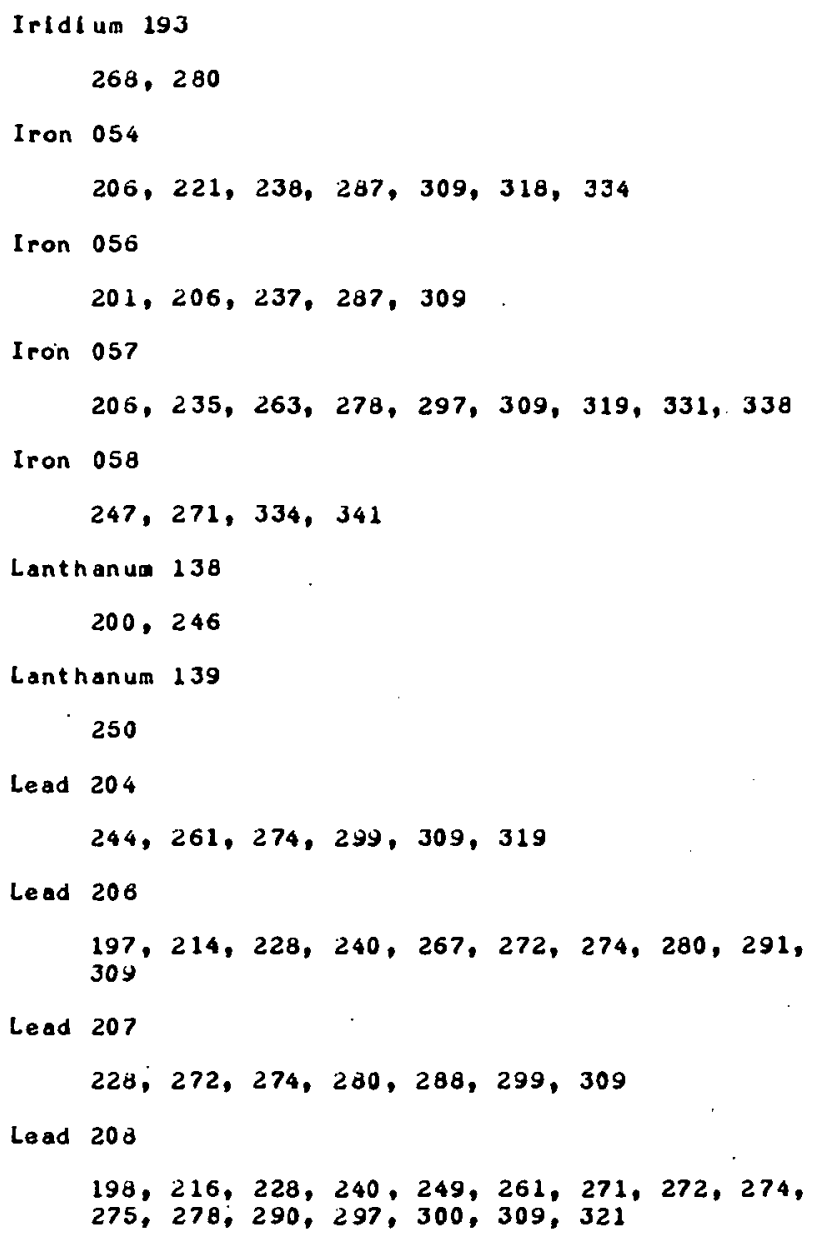

Li thi um 006

$199,204,209,222,241,269,271,273,275$, $289,290,294,306,309,313,319$

Lithi un 007

$204,209,214,249,259,273,278,280,306$, 309,329

Lutet tuas 175

$203,2 \leq 0$

Lutetiun $17 \epsilon$

$200,250,309$

Magnesium 0.24

$304,214,230,251,265,272,282,287,309$

Magneslum 025

$201,204,223,230,251,272,282,287,309$, 319,332

Magneslum 026

$201,204,243,250,251,261,265,269,271$, $272,282,309$

Mercury 196

$247,250,258,29 \%$

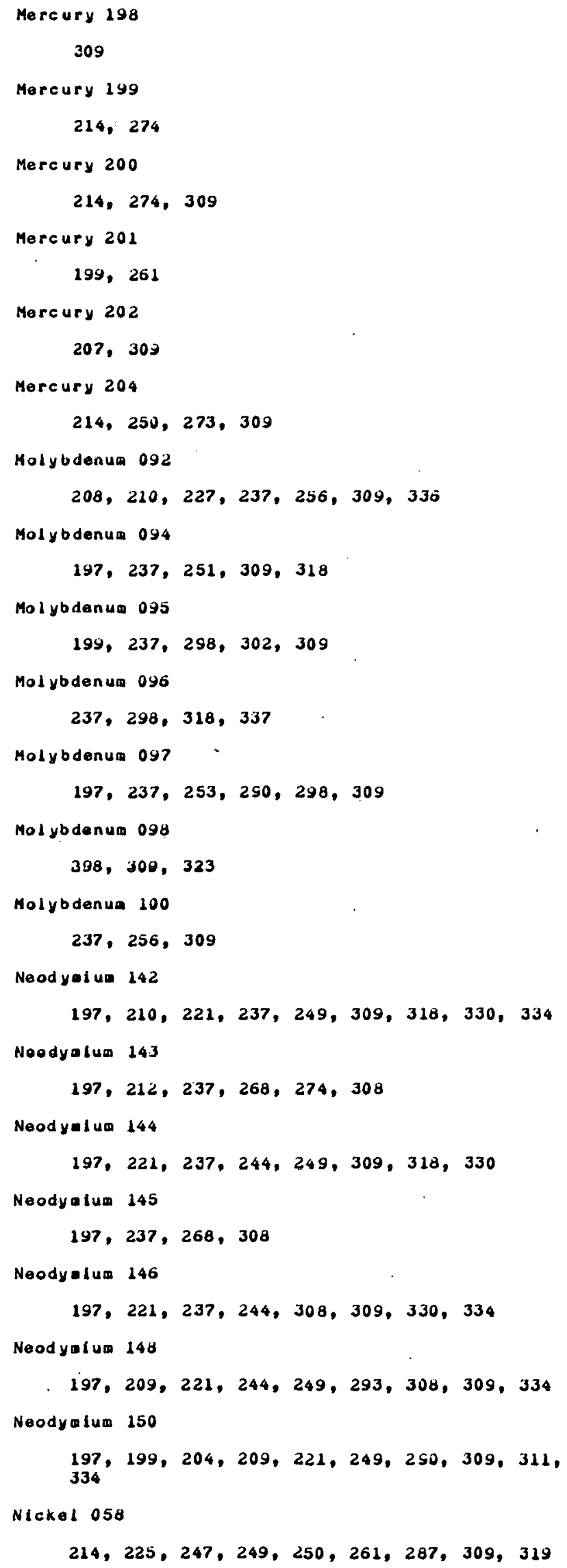


Nicke 1060

$214,225,237,250,261,309$

Nickel 061

$261,276,309,319,334,338$

Nlckel 062

$197,237,247,249,251,261,304,309,334$

Ni ckel 064

$225,231,237,249,261,272,287,309,334$

Osmium 186

204

Osmlum 187

209

Domiun 180

225

Osmlum 189

225.309

Dsmí um 190

$204,225,250$

Comi um 192

$217.225,244,253$

Palladium 104

$199,237,309,321$

Palladium 105

261.290

Pallauluil 100

$199,237,250,290,305$

Palladium 108

$199,204,237,250,252,272,274,309,319$

Palladiun il 10

$199,209,214,237,274,275,287,290,309$

Platinum 192

260,290

Plat I num 194

$230,212,218,284,290,2 \$ 8,309,315$

Plat Inum 195

261,290

Plati num 196

$201,217,261,278,290,309,315,321,325$

Potasslum 039

249,262

Potassium 040

253
Potassium 041

$214,231,249,250,262,314,327,328$

Rhentua 185

254. 309

Rhen fum 187

309

RubldI us 085

314

Rubldium 087

$200,274,284,314,328$

Kuthenium 096

$214,224,244,251,334$

Rullienlum osd

$224,251,320$

Ruthenium 099

$224,242,290,309$

Rutheniun 100

$224,237,280,309$

Ruthenlum 101

237,280

Ruthenlum 102

$218,224,237,280,286,309$

Ruthenlum 104

$224,237,250,280,286,309$

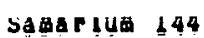

$199,220,221,236,237,244,249,262,260$, 287, 309

Samarium 147

237. 244. 249, 268

Samarlua 148

$199,221,287,290,509,338$

Samarlum 149

$199,237,244,266$

Samarlue 1 sin

$199,237,249,262,301,309,319,334$

Samerium 152

$222,244,276,287,301,309$

Sagarlum 154

$199,209,244,250,262,268,309$

Selentum 074

$224,242,247,258$

Selentum 076

$214,221,298,334$ 
Selenium 077

221,223

Selenium 078

$199,214,221,2 ¥ 8,334$

Selen I um 080

$199,221,309,354$

Selenium 082

$199,214,221,309$

silicon 028

$226,237,249,286,290,304,309$

silicon 029

$$
249,276,290,309
$$

sillicon 030

$199,237,249,250,273,290,293,306,309$

Silver 107

$227,237,309,326$

Silver 109

$197,237,250,251,309,326$

Speclal Services

$199,201,206,207,211,214,221,224,226$

$239,246,247,250,258,261,265,266,271$

$272,273,287,290,302,306,308,309,318$,

$319,327,331,341$

Strontium 084

$250,258,271,297,309,314$

Strontl um 086

214

Strontium 087

250,310

Stron tl um 088

$247,250,300,309,315$

sulfur 032

290

Sulfur 033

$253,276,309$

suitur oja

$201,257,261,270,290,298,306,309$

Tantal um 180

318

Target preparation Stable

$199,201,204,206,211,214,221,224,227$

$228,237,245,250,255,256,260,262,267$,

$271,272,275,278,282,285,286,287,290$,

$293,257,303,309,310,315,319,324,336$,

339,340
Tellurlum 120

209,287

Tollurium 122

$237,247,264,287,309,330$

Tellurlum 123

229

Telluriug 124

$209,219,237,250,253,271,280,287,309$, 319

Tellurlum 125

$223,229,267,298,309$

Tellurlum 226

$237,287,290,319$

Tellurlum 128

$209,251,267,287,240$

Tellurlum 130

$250,267,287,290,341$

Thatllum 203

$247,267,309,317$

Inat1lum 205 .

290,309

Iin 112

211, 247, 250, 267, 309

Iin 114

267,309

Iin 115

309

Tin 116

$199,250,309$

Tin 117

$226,267,309$

I in 118

$$
227,267,309
$$

Tin 119

$$
217,226,249,267,376,309
$$

Iin 120

$$
199,231,237,290,309
$$

Tin 122

$199,220,231,249,309$

Tin 124

$220,250,309$

Titanium 046

$239,245,250,287$ 
Ii tanium 047

239,250

$T 1 \tan \{$ um 048

$224,239,250,287,309,339$

It tanl un 049

$239,250,303,310,318$

$I I \tan i$ um 050

$201,221,239,245,287,309,335,339$

Tungsten 180

$290,318,324$

Tungsten 102

$273,300,305$

Tungetan 163

308,309

Tungsten 184

$273,290,309$

Tungsten $18 \epsilon$

$253,273,290,309$

Ytterbium 168

$247,258,280,319$

Ytterblua 170

$214,251,252,290$

Ytterblum 171

$246,250,251$

Yterbluu 172

$214,221,250,290$

Ytterbium 174

214

Ytterblum 176

$214,221,237,304,314,314$

21 nc 064

$221,265,278,290,298,309,340$

Zlne 066

298, 309

2 inc 067

$250,253,309$

$2 i$ nc 068

261,290

21 ne 070

287

21 rconium 090

$214,250,285,290,308,309,318$
Zlrcontum 091

$214,268,274,290,309$

$2 i$ rconium 092

$214,221,226,290,308,309,312,324$

Zircontum 094

$214,250,290,309,324$

Zirconlum 090

$214,250,271,272,309,340$ 
III. ALPHABETICAL LIST OF STATES AND COUNTRIES, CROSS-REFERENCED TO CUSTOMER NUMBERS

A. DOMESTIC

Al abama

4. 5

Al ask a

6

Arlzona

11

Arkangas

12

Callitornia

$14,24,25,26,27,28,29,30,31,32,33$,

$34,35,42,54,61,67,73,77,80,86,95$,

$102,131,138,144,148,150,151,152,260$,

172,181

Colorado

$2,45,46,47,49,166$

Connecticut

$50,55,120,176,195$

District of Columbia

$37,39,79,111,146,167,173$

Florida

$63,64,65$

Oeviyla

$62,70,71,72$

Idaho

3

Illinols

$10,40,83,83,103,121,123,175$

Indlana

$84,124,137$

Iowa

88,93

Kansas

91

Kenluck y

92

Louls i an a

94,165

Maryl and

$15,90,97,164,170,174$

Massachuset

$43,75,98,99,100,112,177,194$
Michlgan

$66,78,85,105,106,188$

Ainne sot a

109, 122

Hissourl

96,110

Naw Jersey

$8,18,103,113,136,143,154$

New Mexico

$36,114,145$

New York

$13,21,22,48,51,52,53,60,68,76,87$, $89,125,116,117,141,153,161,190,146$

North Carolina

$58,118,119$

Ohio

$41,74,81,104,126,127,128,157,169$ Ok l ahoma

59

One Tlae Billings

180

Dregon

$129,130,139$

Pennsyluanta

$16,38,133,134,135,142,159,289,191$

Rhode IsI and

23,140

South Carolina

$9,57,147$

Tennessee

$125,155,162,163,179$

Texa $\mathbf{3}$

$1,17,20,56,149,156$

Ut ah

19. 178

Virglnia

$44,69,168,171,182,183,184$

Washlngton

$107,132,185,186,192$

west VIrginia

158, 187

wi sconsin

$7,101,193$ 


\section{B. FOREIGN}

Argentina

197

Augtralia

$198,199,200,201,202,203$

Austria

204

Be lgi um

$205,206,207,208,209$

Brazl I

$210,211,212$

Canad a

$213,214,215,216,217,218,219,220,221$, $222,223,224,225,226,227,228,229,230$, 231,232

Denmark

$233,234,235,236,237$

England

$238,239,240,241,242,243,244,245,246$, $247,248,249$

France

$250,251,252,253,254,255,256,257$

Germany

$258,259,260,261,262,264,265,266,267$,

$268,269,271,272,273,274,275,276,277$,

$278,279,280,281,282,283,285,286,287$,

$288,289,290,291,292,293$

Indla

397,289

Israel

$299,300,301$

Italy

$302,303,304,305,308,307$

Japen

$308,309,310,312$

Korea

313

Mexico

314

Ne the $r$ lands

$315,317,318,319,320,321$

New Jersey

311

New Zeal and
Norway

323

Scot land

324,325

South Africa

$326,327,328$

Sweden

$329,330,331,332,333,334$

Switzerland

$335,336,337,338,339,340$

The Netherlands

316

Turkey

341

Wost Gormany

$263,270,284,294,296,236$ 
ISOTOPE

ANT I MONY-1 21

ANTI MONY -123

BARIUM- 1 J0

BARI UM-130

BARIUM-13S

BARIUM-137

ARIUM-137

BARIUM-13

GORON-11 (DURIC)

BORON-11 ELEMENT

BROM INE 79

BR OMINE -81

CADM IUM-1 106

CADM IUM-108

CAOMIUM-110

EADM IUM-111

EAOM IUM-112

CADM IUM-11 4

ADM IUM-116

CALC IUM-40

CALCIUM-42

CALCIUM-43

CALCIUM-40

CALCIUM-46

CARBON-12

CERIUM-136

CERI UM-1 38

CERIUM- 140

CERIUM-142.

CHLORINE-35

CHROMI UM-5O

CHROMIUM-52

CHROMI UM- 53

CHROMI UM-54

COPPER-63

OY SPROS IUM-15

OY SPROS I UM-158

OYSPROSIUM-160

OY SPROSIUM-161

OY SPROS IUM-1 63

OYSPROSI UM- 164

ERBIUM- 162

ERBIUM-164

ERBIUM-160

ERBIUM-167

ERBIUM-168

EUROPIUH-151

EUROPIUM-15

GADOLINIUM-15 SHIP D O MEESTIII C DOLLARS SHIP

ORE I G N
MILLI I RAMS

Dolars SHIP PROJECT

DOLLARS. SHIP

TOTAL

\begin{tabular}{|c|c|c|}
\hline 132.0 & 205 & $i$ \\
\hline 16.0 & 384 & 2 \\
\hline 204.0 & 6120 & 1 \\
\hline 271.0 & 650 & 6 \\
\hline 217.0 & 413 & 7 \\
\hline 180.0 & 252 & 8 \\
\hline 100.0 & 105 & 4 \\
\hline 663.0 & 100 & 2 \\
\hline 2426090.0 & 21774 & 24 \\
\hline 133000.0 & 4846 & 11 \\
\hline 40.0 & 128 & 3 \\
\hline 120.0 & 372 & 5 \\
\hline 50.0 & 995 & 6 \\
\hline 10.0 & 162 & 2 \\
\hline 915.0 & 001 & 5 \\
\hline 629.5 & 410 & 5 \\
\hline 4000.0 & 1800 & 5 \\
\hline 1356.0 & 1029 & 4 \\
\hline 81635.0 & 32700 & 6 \\
\hline 29000.0 & 7250 & 5 \\
\hline 261.0 & 2218 & 6 \\
\hline 9.0 & 522 & 6 \\
\hline 1838.7 & 4174 & 5 \\
\hline 6.0 & 3514 & 6 \\
\hline 751.5 & 22858 & 16 \\
\hline 50.0 & 215 & \\
\hline & & 1 \\
\hline 330.0 & 50 & 7 \\
\hline 190.0 & 260 & 8 \\
\hline 1655.0 & 827 & 6 \\
\hline 10.0 & 62 & 9 \\
\hline 1461.0 & 3559 & 9 \\
\hline 562.0 & 309 & 8 \\
\hline 55.5 & 50 & 3 \\
\hline 100.0 & 625 & 4 \\
\hline 5720.0 & 1169 & 12 \\
\hline 2423.0 & 869 & 9 \\
\hline 1.5 & 50 & 1 \\
\hline 375.0 & 150 & 3 \\
\hline & & 4 \\
\hline & so & 1 \\
\hline 50.0 & 450 & 3 \\
\hline 50.0 & 187 & 4 \\
\hline 416.5 & 300 & 3 \\
\hline 367.0 & 520 & 2 \\
\hline 50.0 & 237 & 10 \\
\hline 140.0 & 100 & 2 \\
\hline 333.0 & 150 & 9 \\
\hline 250.0 & 125 & 3 \\
\hline 160.0 & 464 & 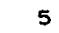 \\
\hline
\end{tabular}

$$
\begin{array}{r}
50.0 \\
50.0 \\
40.0 \\
10.0 \\
430.0 \\
442.0 \\
621.7 \\
515.0 \\
665.0 \\
611900.0 \\
5000.0 \\
49500.0 \\
165.0 \\
455.0 \\
162.5 \\
40.0 \\
329.0 \\
1530.0 \\
1530.0 \\
467.0 \\
1750.0 \\
350.0 \\
1900.0 \\
365.0 \\
202.0 \\
241.7 \\
65.0 \\
435.0 \\
\\
10.0 \\
110.0 \\
2133.0 \\
725.7 \\
2875.0 \\
703.0 \\
2970.0 \\
1661.0 \\
364.0 \\
170.0 \\
5420.0 \\
3632.0 \\
197.0 \\
255.0 \\
680.0 \\
450.0 \\
1125.0 \\
492.8 \\
200.0 \\
137.0 \\
350.0 \\
600.0 \\
610.0 \\
3650.0 \\
200.0 \\
2790.0 \\
700.0 \\
240.0 \\
\end{array}
$$

mily zGrams dollatas

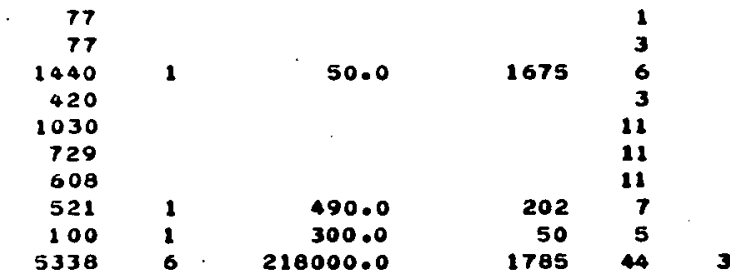

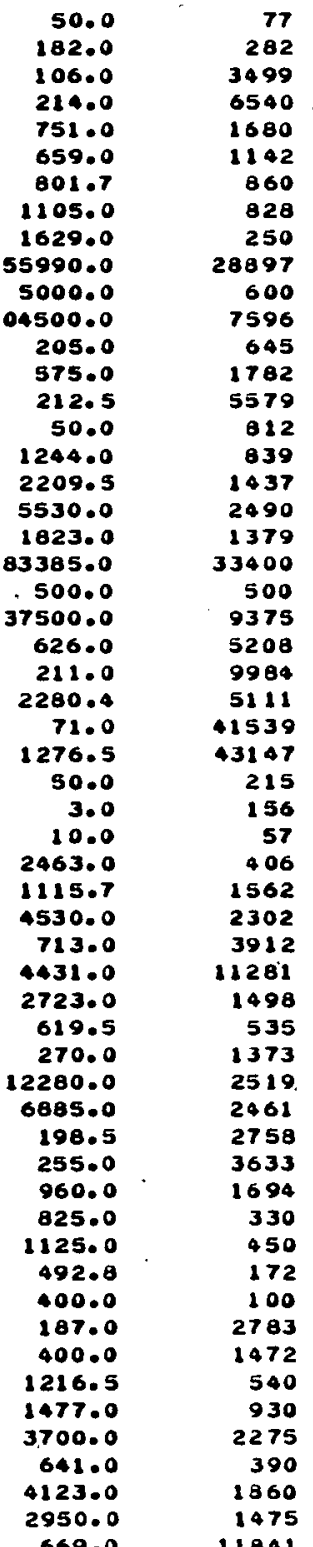


P R O D. E C T MILLIGRAMS DOLLARS SHIP

TOTAL

GADOL INIUM-154

GADOL INIUM-156

GADOLI NI UM-157

GADOL INIUM-158

GAOOLINIUM-160

GALL IUM- 69

GERMANIUM $\rightarrow 0$

GERMANIUM -72

GERMANIUM-?4

GERMANIUMTTS

H.P. GAOUL INIUM

HAFNIUM-17C

HAFNIUM-17T

MAFNIUM -178

HAFNIUM-179

INDI UM- 113

INDIUM-1:5

IR IOIUM-191

IR IOIUM-
IR ON-S4

IRON-56

IRON-57
IRON-58

LANTHANUM-138

LANTHANUM-139

LEAO-204

LEAO-206

LEAD-208

LITHIUM-ó

LITHIUM-7

LUTETIUM-175

LUTETIUM-176

MAGNES IUM-24

MAGNES IUM-26

EERCURY-196

MERCURY - 198

MERCURY-199

MERCURY- 200

MERCURY - 201

MERCURY-202.

MOL'YBDENUM-U92

MOLY YDENUM - 094

MOL YODENUM -094

MOL YBDENUM-096

MOL YBDENUM-097

MJLL YBDENUM-098

MOL YBDENUM-1 00

NEODYMIUM-1 72

NEODYMIUM- 143

\begin{tabular}{|c|c|c|c|}
\hline 1 & 30.0 & 307 & 4 \\
\hline 3 & 119.0 & 655 & 2 \\
\hline 1 & 56.0 & 50 & 5 \\
\hline 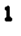 & 100.0 & 100 & 1 \\
\hline$?$ & 3400.0 & 1700 & 9 \\
\hline & & & 1 \\
\hline 2 & 400.0 & 150 & 5 \\
\hline 3 & 475.0 & 162 & 3 \\
\hline 2 & 500.0 & & 6 \\
\hline 3 & $22 \mathrm{a} .0$ & 608 & 4 \\
\hline & & & 1 \\
\hline & & & 2 \\
\hline & & & 1 \\
\hline 1 & 100.0 & 50 & 2 \\
\hline 3 & 96.0 & 150 & 2 \\
\hline 1 & 260.0 & 130 & 5 \\
\hline 2 & 85.0 & 1020 & \\
\hline 2 & 1200.0 & 540 & 1 \\
\hline 1 & 15.0 & 75 & 3 \\
\hline 1 & 10.0 & 50 & 2 \\
\hline 5 & 3044.0 & 4567 & 8 \\
\hline 7 & 11500.0 & 1150 & 7 \\
\hline 2 & 1293.0 & 3098 & 15 \\
\hline 4 & 478.0 & 8210 & 3 \\
\hline 1 & 3.0 & 129 & 2 \\
\hline 3 & 143.0 & $10 ? 9$ & 1 \\
\hline 2 & $100-0$ & 60 & $\therefore$ \\
\hline & 100.0 & 60 & 14 \\
\hline & 100.0 & 165 & 9 \\
\hline & 6710.0 & 1202 & 25 \\
\hline 3 & 9158000.0 & 13586 & 22 \\
\hline 3 & 398360503.0 & 154619 & 19 \\
\hline 1 & 550.0 & 192 & 2 \\
\hline 3 & $7 \cdot 0$ & 301 & 3 \\
\hline 0 & 1650.0 & 607 & 14 \\
\hline 8 & 340.0 & 850 & 13 \\
\hline 9 & 3129.0 & 4544 & 17 \\
\hline & & & 5 \\
\hline 3 & 70.0 & 770 & 1 \\
\hline 5 & 235.0 & 1527 & 2 \\
\hline 3 & 160.0 & 640 & 4 \\
\hline 2 & 40.0 & 510 & 1 \\
\hline 3 & 145.0 & 2320 & 2 \\
\hline 3 & 75.0 & 750 & 6 \\
\hline 1 & 1000.0 & 300 & 9 \\
\hline 2 & 256.5 & 100 & 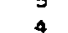 \\
\hline 1 & 100.0 & 50 & 3 \\
\hline 1 & .111 .0 & 50 & 6 \\
\hline 1 & 150.0 & 150 & 7 \\
\hline 2 & 500.0 & 250 & 6 \\
\hline 3 & 490.0 & 338 & 13 \\
\hline & & 585 & 2 \\
\hline
\end{tabular}

$\begin{array}{rr}1569 & \\ 1250 & \\ 424 & \\ 50 & \\ 815 & 4 \\ 949 & 7 \\ 50 & 1 \\ 200 & 1 \\ 331 & 2 \\ 251 & 1 \\ 770 & \\ 380 & 3 \\ 910 & 2 \\ & 2\end{array}$

6.0
350.0

100.0

200.0

150.0
800.0

1505

110

232

111.0

190.0

620.0

6065.0
310.0

310.0

200.0

335.0

7836.0
155089.0

7710895.0

7710895.0
31557054.0

236.0

27.0
7543.0

1872.0

4885.0

180.4

140.0

270.0

60.0

300.

769.5
730.0

48350.0

570.0

3596.0

5
95
20
209
85
1434
785
64
20
225
577
339
1692
1385
1430
100
116
268
4682
711
7048
880
910
1080
25
960
277
455
429
45
1147
474
4835
400
2239
509

630.0
630.0
3330.0
500.0
50.0
95.0
100.0
265.0
120.0
25.0

567
346
1858
275
50
100
50
150
264
256

225.0

719.0

130.0

2070.0

8834.0

590.0

250.0

1490.0

410.0

2565.0

25.0

6.0

350.0

100.0

300.0

246.0

85.0

1311.0

380.0

160.0

24386.0

24386.0
7358.0

845.0

21.0
200.0

578.0
8184.0

8061.0

76914.0

18567929.5

15115.0

1699034.5

367004.5
4293.0

293.0
2.0
570.0

570.0
660.0

660.0

10.0

50
1811

2982

1203

86
275

275
1675

4832

110

5129.0

36.0

9763.0

2872.0
14084.0

180.4

160.0

375.0

430.0

205.0
415.0

2569.5

$\begin{array}{r}730.0 \\ \hline\end{array}$

4271.5

14500.0

3264.0

4711.0

4711.0
1215.6

1905

1041

150
1161

4507

325

250
581

463
770

$\begin{array}{r}1782 \\ \hline\end{array}$

256
57

1505

150

382

530
1020

590

1900

0087

2526

17547

903

200

3558

59.56

19940

30422

158485

1495

1548
3567

7207

16494

1760

2437

1720

765

3280
3925

795

428
1257

2758

2758
1469

1469
48502

3824

2987
1094 
ISOTOPE

SHIP O OMESTIC

NEOOYMIUM-144

NEOOYMIUM-145

NEDOYMIUM-150

NICKEL-5O

NICKEL -62

NICKEL -64

OSMIUM- 180

OSMIUM- 188

OSNIUM-1 39

OSM I UM-190

OSMIUM- 192

PALL AD I UM 102

PALLADIU:M- 104

PALLADIUM-105

PALLADIUM- 106

PALLADIUM-108

PALL AOI UM-110

PLATINUM-192

PLATINUM-194

PLATINUM-195

PL AT I NUM-196

potassIUna-39

POTASSI UM-4

RHENIUM-1 85

RHENIUM-1 \&7

RUBI DIUM-85

RUBIOIUM-87

RUTHENIUM-098

RUTHENIUM-099

RUTHENI UM- 100

RUTHENIUM-102

RUTHENIUM- 104

SAMARI UM-1 44

SAMARI UM-147

SAMAR IUM-1 4

SAMARI UM-1 49

SAMARIUM-15O

SAMARI UM-1 52

SAMARIUM-154

SELENIUM-7 6

SELENIUM -77

SELENIUA -78

SELENIUM-BO

SELE NI UM-B2

SILICON-28

SILICON-29

SILICON-30

SILVER-107
IC F OREIG

\begin{tabular}{|c|c|}
\hline $\begin{array}{r}16540.0 \\
870.0\end{array}$ & $\begin{array}{l}4962 \\
1087\end{array}$ \\
\hline 3998.0 & 1455 \\
\hline 225.0 & 250 \\
\hline 380.0 & 572 \\
\hline 5233.0 & 825 \\
\hline 3800.0 & 760 \\
\hline 50.0 & 262 \\
\hline 17979.0 & 30435 \\
\hline 45.0 & 900 \\
\hline 200.0 & 550 \\
\hline 72.0 & 162 \\
\hline 1893.5 & 3030 \\
\hline 100.0 & 95 \\
\hline 17.0 & 935 \\
\hline & \\
\hline 470.0 & 800 \\
\hline 255.0 & 944 \\
\hline 110.0 & 110 \\
\hline 200.0 & 550 \\
\hline 735.0 & 1470 \\
\hline 1030.0 & 826 \\
\hline 900.0 & 4500 \\
\hline 76.0 & 50 \\
\hline 125.0 & 50 \\
\hline 690.0 & 380 \\
\hline 1059.8 & 3605 \\
\hline 20.0 & 200 \\
\hline 20.0 & 76 \\
\hline 20.0 & 105 \\
\hline 20.0 & 60 \\
\hline 130.0 & 205 \\
\hline 170.0 & 442 \\
\hline 800.0 & 660 \\
\hline 171.0 & 51 \\
\hline 125.0 & 50 \\
\hline 1019.0 & 371 \\
\hline 200.0 & 100 \\
\hline 2488.3 & 394 \\
\hline 300.0 & 100 \\
\hline 342.0 & 39330 \\
\hline 266.0 & 506 \\
\hline 564.0 & 1184 \\
\hline 475.0 & 641 \\
\hline sio.o & 460 \\
\hline 521.0 & 1093 \\
\hline 600.0 & 690 \\
\hline 312.0 & 1638 \\
\hline 1543.0 & 6474 \\
\hline 5754.3 & 3057 \\
\hline
\end{tabular}

4630.0
350.0
2265.0
1375.0
8200.0
5693.0
4570.0
460.0
4635.0
450.0
183.0
100.0
20.0
122.0
666.0
362.0

320.0
400.0
465.5
1701.0
1970.0
70.0
800.0
250.0
1350.0
1300.0
200
1105.0
228.0
120.0
100.0
430.0
150.0
60.0
630.0
240.0
70.0
367.0
400.0
3942.0
1238.0
1160.0
692.0
1050.0
4293.0
2571.0
220.0
206.0
73.0
330.0
293.0
254.0
1579.0
030.0
1143.0
1460.0

p o o J E T doLlars SHIP'

TOTAL DOLARS SHIP

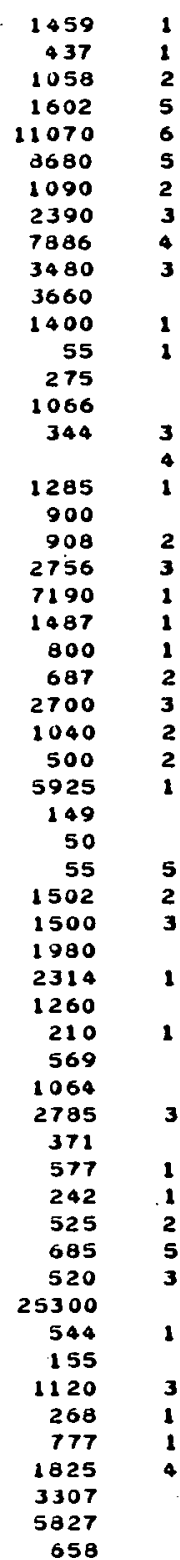

200.0
100.0
400.0
1315.0
405.0
14480.0
205.0
2023.0
1443.0
1000.0
10.0
10.0
100.0

1700.0
18.0
20.0
81.0
903.0
225.0
100.0
20.0
54.0
749.0
250.0
250.0
41.0
200.0

4590.0
120.0
110.0
1100.0
1229.0
473.0
75.0
58.0
1171.0
200.0
100.0
300.0
1450.0
145.0
100
100.0
1100.0
100.0
50.0
1285.0

milligaAms dollars

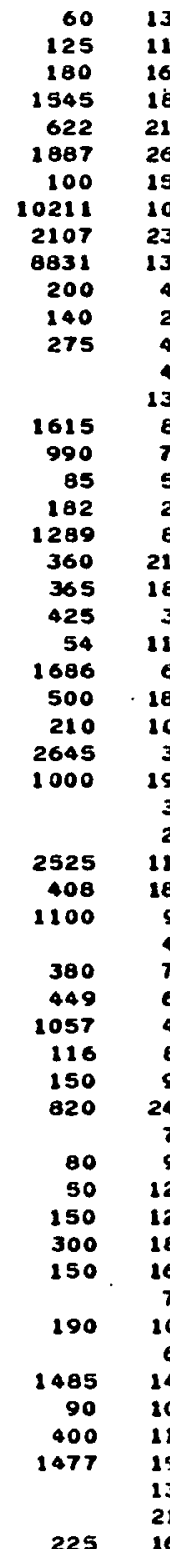

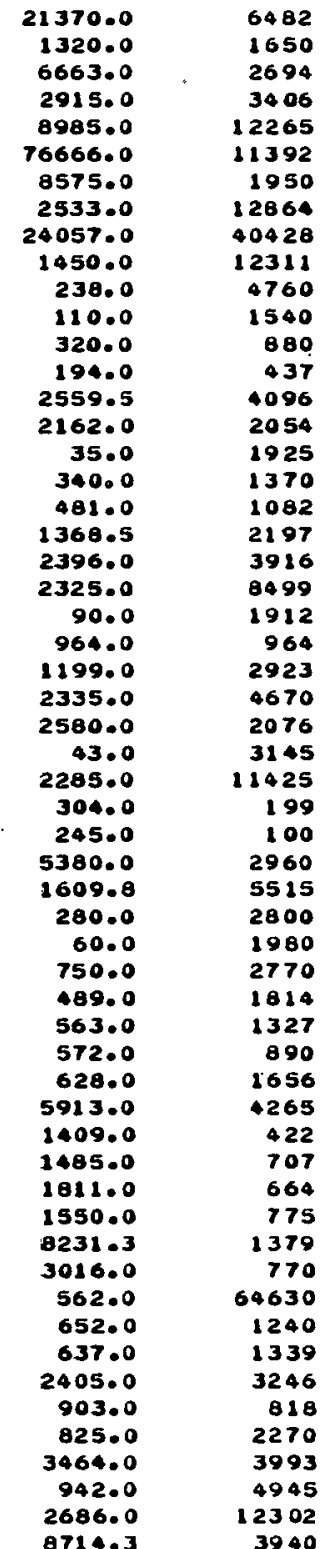


D OAESTI =

SHIP

SILVER-109

STRONTI UM-B

STRONTIUM-BO

STRONT I UM-87

STRONTIUIM-OE
SULFUR-32

SULF UR-33

TANTALUM-1 80

TANTALUM-180

TELLURIUMA- 122

TELLLURI UM- 123

TELLURIUM-124

TELLURI UM-125

TELLURI UM-128

TELLURIUM- 130

THALL IUM-2U3

THALL I UM-205

TIN-112

TIN-114

IIN-116

TIN-117

$\operatorname{TIN}-118$

TIN-120

TIN-122

TIN-124

TITANIUM-40

TITANI UM-A?

TITANIUM-48

TITANI UM-49

TEN-180

TUNGSTEN-180

TUNGSTEN-1OL

TUNGSTEN-1 83

TUNGSTEN-1.04

TUNGSTEN-186

YTRERBIUM-168

YTTERBIUM-17

YT TERBI UM-172

YTTERBIUM-1 73

YTTERBI UM-1 78

ITTERAIUM-176

ZI NC-64

ZINC-66

ZINC-67

$\operatorname{ZINC}-68$
$\operatorname{ZINC}-70$

ZIRCONIU:A-90

ZIRCONIUM-9

ZIRCONI UM -92
ZIRCONIUM -94

ZIRCONIUM- -96

Lans

TOTAL

$800 \quad 01.041629 .4$

\begin{tabular}{|c|c|c|c|}
\hline 2 & 810.0 & 486 & 7 \\
\hline 3 & 103.0 & 188 & 8 \\
\hline 6 & 3700.0 & 1850 & 1 \\
\hline \multirow[t]{2}{*}{1} & 71.0 & 50 & 2 \\
\hline & 5559.0 & 277 & 6 \\
\hline 3 & 250.0 & 250 & i \\
\hline 4 & 53.0 & 1930 & 3 \\
\hline 4 & 117.0 & 944 & 8 \\
\hline 8 & 7072.4 & 47733 & ${ }_{i}^{2}$ \\
\hline 6 & $10076-1$ & & 1 \\
\hline 4 & 294.0 & 338 & 5 \\
\hline 2 & 584.0 & 350 & 4 \\
\hline 6 & 1016.0 & 360 & 5 \\
\hline 5 & 1320.0 & 455 & 8 \\
\hline 10 & 140210.0 & 77170 & 6 \\
\hline 4 & 3300.0 & 660 & 2 \\
\hline 1 & 3.0 & 60 & 7 \\
\hline 1 & 7.5 & 65 & 2 \\
\hline$\dot{a}$ & 1100.0 & 330 & 4 \\
\hline 3 & 450.0 & 225 & 3 \\
\hline 2 & 342.5 & 120 & 4 \\
\hline 5 & 1050.0 & 382 & 10 \\
\hline 3 & 1400.0 & 350 & 5 \\
\hline 2 & 500.0 & 325 & 7 \\
\hline 5 & 1950.0 & 1072 & 5 \\
\hline 1 & 50.0 & 70 & 3 \\
\hline 3 & 134.0 & 181 & $i$ \\
\hline 4 & 1285.0 & 257 & 7 \\
\hline 4 & 657.0 & 1511 & 5 \\
\hline 2 & 57.0 & 100 & 7 \\
\hline 4 & 206.0 & 2250 & 3 \\
\hline 4 & 2800.0 & 700 & 4 \\
\hline 2 & 610.0 & 366 & 1 \\
\hline 2 & 3200.0 & 960 & 3 \\
\hline 2 & 500.0 & 175 & 4 \\
\hline 3 & 27.0 & 479 & 11 \\
\hline 3 & 860.0 & 2720 & 4 \\
\hline 3 & 252.0 & 151 & 3 \\
\hline 1 & 550.0 & 220 & 4 \\
\hline 2 & 750.0 & 300 & \\
\hline 1 & 874.0 & 218 & 1 \\
\hline$i$ & 170.0 & 59 & 6 \\
\hline 5 & 953.0 & 335 & 7 \\
\hline 7 & 850.9 & 468 & 2 \\
\hline 10 & 535.5 & 1985 & 3 \\
\hline 10 & 13670.0 & 10936 & 1 \\
\hline 5 & 41.0 & 1271 & 1 \\
\hline 2 & 225.0 & 100 & 4 \\
\hline$i$ & 48.0 & 50 & a \\
\hline 1 & 500.0 & 475 & 8 \\
\hline 2 & 35.0 & 150 & 6 \\
\hline
\end{tabular}

ORE I GN

DOLARS SHIP PR O JECT

MILLIGRAMS DOLLARS SHIP

TOTAL

MILLIGRAMS DOLLARS

1054.3

326.0

$\$ 00.0$

14300.0

100.0

35.0

272.0

10.0
25.0

1930.0

50.0
6505.0

950.0

350.0

11450.0

112100.0

1100.0

1100.0
4133.0

540.0

20.0
3531.0

600.0

5335.0

4140.0

1200.0

3500.0

403.0

618.0

5964.0

3265.5

21.0

800.0

80.0
565.0

565.0
740.0

740.0
405.0
620.0

620.0

145.0
1175.0

1000.0

1832.8
1532.0

1532.0
180.0

133.0

100.0

50.0
3340.0

1350.0

462.5

190.0
215.0

532
13173

$\begin{array}{ccc}400 & 4 & 450.0 \\ 525 & 1 & 100.0 \\ 775 & 4 & 1600.0 \\ 100 & & \vdots\end{array}$

350
2375

13027

13027
150

24719

1002
230

610
2914

61655

250
94025
4725

4725
300

1060
300

1905
1255
300

300
635
1925

1925
561
034

034
1263

1263
4836

5303
1427

1427
200
50

50
170

260
14252

2603

150
510

150.0

1850.0

500.0

100.0

400.4
400.9

200.0

1288.0
50.0

150.0

4467.0

50.0

$\$ 2200.0$

3200.0

750.0

330.0
4484.0

40.0

200.0

100.0

50.0

500.4

250
642

547
100

90 32285.

1550

880
1417

524

1022

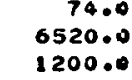

44.0

6499441203

40581751.5

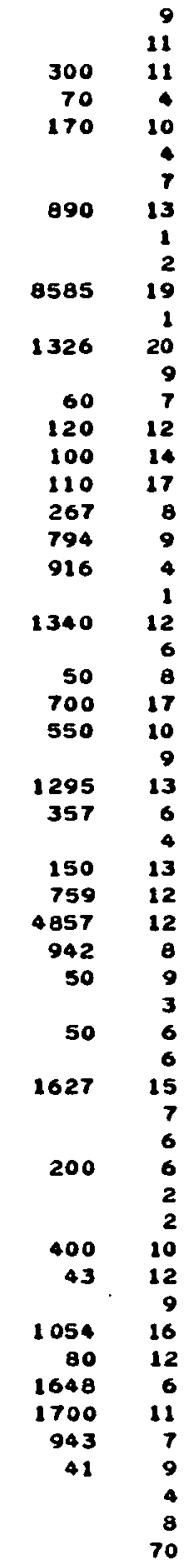

1864.3

$4950.0 \quad 2550$

921.0

21459.0

350.0
88.0

83.0
539.0

10.0

10852.4

50.0

17041.0

1244.0

1034.0

3333.0

52510.0

4186.0

697.5

20.0

9098.0

1050.0

5727.5

9190.0

4800.0

14550

708.0

752.0

7999.0

3169.0

7806.5

233.0

3800.0

690.0
3865.0

3865.0
1240.0

562.0

1480.0

397.0
2225.0

2225.0
750.0

1874.0

3022.8

2663.0

1030.9

954.5

1385.0
16850

0085.0

2590.0

2618031.

$1291762376 \quad 656703727.2$

1545842 
ORNL/TM-6241

INTERNAL DISTRIBUTION

$\begin{aligned} \text { 1. } & \text { W. A. Bell, Jr. } \\ 2 . & \text { K. B. Brown } \\ 3 . & \text { S. E. Curtis } \\ 4-5 . & \text { W. C. Davis } \\ 6 . & \text { D. E. Ferguson } \\ \text { 7. } & \text { H. R. Gwinn } \\ 8 . . & \text { J. E. Keeton } \\ 9 . & \text { E. Lamb } \\ \text { 10. } & \text { C. L. Ottinger } \\ 11-15 . & \text { E. Newman } \\ 16 . & \text { F. G. Perey } \\ 17 . & \text { H. Postma } \\ 18 . & \text { W. K. Prater } \\ 19 . & \text { W. R. Ragland } \\ 20 . & \text { M. E. Ramsey }\end{aligned}$
21.
22.
J. E. Ratledge
23.
J. G. Tracy
B. Trauger
24. R. G. Wymer
25 .
A. Zucker
26.
27.
G. R. Choppin (consultant)
E. L. Gaden, Jr. (consultant)
28. C. H. Ice (consultant)
29. L. E. Swabb, Jr. (consultant)
30. K. D. Timmerhaus (consultant)
31. Laboratory Records - RC
32-41. Laboratory Records
42. ORNL Patent Section
45. Document Reference Section

43-44.

\section{EXTERNAL DISTRIBUTION}

46. J. L. Burnett, Jr.,, Division of Physical Research, DOE, Washington, DC 20545

47. J. A. Lenhard, Research and Technical Support Division, DOE-ORO

48. J. N. Maddox, Division of Biomedical and Environmental Research, DOE, Washington, DC 20545

49. J. W. Nehls, Research and Technical Support Division, DOE-ORO

50. H. S. Oster, Jr., Research and Technical Support Division, DOE-ORO

51. G. L. Rogosa, Division of Physical Research, DOE, Washington, DC 20545

52. R. H. Schoonmaker, Office of the Controller, DOE, Washington, DC 20545

53. R. A. Schwind, Monsanto Research Corporation, Miamisburg, $\mathrm{OH} 45342$

54. J. L. Simmons, Battelle-Pacific Northwest Laboratory, Battelle Blvd., Richland, WA 9935 ?

55. J. S. Kane, Division of Physical Research, DOE, Washington, DC 20545

56. W. W. H. Weyzen, Division of Biomedical and Environmental Research, Washington, DC 20545

57-83. Technical Information Center, Oak Ridge, TN 37830 